LCLS-TN-09-5

\title{
The Physics of the Gas Attenuator for the Linac Coherent Light Source (LCLS)*
}

\author{
D.D. Ryutov, R.M. Bionta, S.P. Hau-Riege, K.I. Kishiyama, D. McMahon, M.D. Roeben, \\ S. Shen \\ Lawrence Livermore National Laboratory, Livermore, CA 94551 \\ P.M. Stefan \\ Stanford Linear Accelerator Center, Stanford University, Stanford, CA 94309
}

\begin{abstract}
A systematic assessment of a variety of physics issues affecting the performance of the LCLS X-ray beam attenuator is presented. Detailed analysis of the gas flow in the gas attenuator and in the apertures is performed. A lot of attention is directed towards the gas ionization and heating by intense X-ray pulses. The role of these phenomena in possible deviations of the attenuation coefficient from its "dialed in" value is evaluated and found small in most cases. Other sources of systematic and statistical errors are also discussed. The regimes where the errors may reach a few percent correspond to the lower X-ray energies (less than $2 \mathrm{keV}$ ) and highest beam intensities. Other effects discussed include chemical interaction of the gas with apertures, shock formation in the transonic flow in the apertures of the attenuator, generation of electromagnetic wakes in the gas, and head-to-tail variation of the attenuation caused by the ionization of gas or solid. Possible experimental tests of the consistency of the physics assumptions used in the concept of the gas attenuator are discussed.

Interaction of X-rays with the solid attenuator (that will be used at higher X-ray energies, from 2.5 to $8 \mathrm{keV}$ ) is considered and thermo-mechanical effects caused by the beam heating are evaluated. Wave-front distortions induced by non-uniform heating of both the solid and the gas are found to be small.

An overall conclusion drawn from the analysis presented is that the attenuator will be a reliable and highly versatile device, provided that some caution is exercised in its use for highest beam intensities at lowest X-ray energies.

\section{Disclaimer}

This document was prepared as an account of work sponsored by an agency of the United States Government. Neither the United States Government nor any of their employees, makes any warranty, express or implied, or assumes any legal liability or responsibility for the accuracy, completeness, or usefulness of any information, apparatus, product, or process disclosed, or represents that its use would not infringe privately owned rights. Reference herein to any specific commercial product, process, or service by trade name, trademark, manufacturer, or otherwise, does not necessarily constitute or imply its endorsement, recommendation, or favoring by the United States Government. The views and opinions of authors expressed herein do not necessarily state or reflect those of the United States Government, and shall not be used for advertising or product endorsement purposes.
\end{abstract}

* This work was performed under the auspices of the U.S. Departent of Energy by Lawrence Livermore National Laboratory under contract No. DE-AC52-07NA27344. Work supported in part by the DOE contract DE-AC0276SF00515. This work was performed in support of the LCLS project at SLAC. 


\section{Table of contents}

$\begin{array}{ll}\text { Basic Notation } & \text { p. } 3\end{array}$

$\begin{array}{ll}\text { 1. Introduction } & \text { p. } 4\end{array}$

$\begin{array}{ll}\text { 2. Beam attenuation } & \text { p. } 6\end{array}$

2.1 Evaluating the attenuation p. 6

2.2 Errors in the attenuation coefficient p. 9

3. Gas flow in the attenuator and the differential pumping system

p. 10

3.1 Gas flow through the apertures p. 10

3.2 Gas flow inside the gas attenuator p. 12

3.3 Gas heating by X-ray pulses p. 14

3.4 Chemical interaction of nitrogen with apertures p. 18

3.5 Possible variation of the attenuation vs. the beam intensity p. 19

3.6 Head-to-tail variation p. 19

$\begin{array}{ll}\text { 4. Thermo-mechanical effects in the solid attenuator } & \text { p. } 20\end{array}$

4.1 Thermal expansion and thermal stresses $\quad$ p. 20

4.2 Effect of the thermal expansion on the beam

4.3 Possible gross deformations of slabs caused by $\begin{array}{ll}\text { thermal stresses } & \text { p. } 25\end{array}$

$\begin{array}{ll}\text { 5. Effect of the attenuator on the wave front } & \text { p. } 26\end{array}$

5.2 Effect of the gas attenuator p. 26

$\begin{array}{ll}5.2 \text { Effect of the solid attenuator } & \text { p. } 28\end{array}$

$\begin{array}{ll}\text { 6. Electromagnetic wake } & \text { p. } 29\end{array}$

7. Calibration of the gas attenuator with respect to the solid attenuator

$\begin{array}{ll}\text { 8. Summary and discussion } & \text { p. } 32\end{array}$

$\begin{array}{ll}\text { References } & \text { p. } 34\end{array}$ 


\section{Basic notation}

\begin{tabular}{|c|c|c|}
\hline Notation & Definition & First introduced \\
\hline$A$ & Attenuation coefficient & Eq. 2.1 \\
\hline$E_{X}$ & Energy of X-ray photons & Table 1; Eq. 3.14 \\
\hline$F$ & X-ray fluence & Eq. 3.15 \\
\hline$L$ & Attenuator length & Eq. 2.2 \\
\hline$M$ & $\begin{array}{l}\text { Total mass of the gas in the } \\
\text { attenuator }\end{array}$ & Eq. 3.7 \\
\hline$N$ & $\begin{array}{l}\text { Total particle inventory in the } \\
\text { attenuator }\end{array}$ & Eq. 3.6 \\
\hline$N_{r}$ & Refraction index & Eq. 5.1 \\
\hline$Q$ & Energy per unit volume & Eq. 3.17 \\
\hline$T$ & Temperature & Eq. 2.5 \\
\hline$V$ & Attenuator volume & Eq. 3.10 \\
\hline$W$ & X-ray pulse energy & Eq. 2.1 \\
\hline$a$ & Radius of an aperture & Fig. 3.1 \\
\hline$b$ & Radius of the attenuator tube & Fig. 3.2 \\
\hline$c$ & Speed of light & Fig. 6.1 \\
\hline$h$ & $\begin{array}{l}\text { Thickness of the solid attenuator } \\
\text { foil }\end{array}$ & Eq. 4.5 \\
\hline$l_{a b s}$ & e-folding attenuation length & Table 1 \\
\hline$n$ & Particle density in gas & Eq. 3.1 \\
\hline$p$ & Gas pressure & \\
\hline$q$ & Energy deposition per unit length & Eq. 3.12 \\
\hline$s$ & Sound speed & Eq. 3.3 \\
\hline$t$ & Time between X-ray pulses & Eq. 3.21 \\
\hline$w$ & Beam radius & Eq. 3.15 \\
\hline$\Sigma$ & Absorption cross-section & Eq. 2.2 \\
\hline$\alpha$ & $\begin{array}{l}\text { Volumetric thermal expansion } \\
\text { coefficient }\end{array}$ & Table 3 \\
\hline$\gamma$ & Adiabatic index & Table 2 \\
\hline$\eta$ & Gas viscosity & Table 2 \\
\hline$\lambda$ & Mean free path & Eq.3.2 \\
\hline$\lambda_{X}$ & Photon wavelength & Eq.5.4 \\
\hline$\rho$ & Mass density & Table 2 \\
\hline$\sigma$ & Poisson ratio & Table 3 \\
\hline$\theta$ & Beam deflection angle & Eq. 5.2 \\
\hline$\chi$ & Thermal diffusivity & $\begin{array}{l}\text { Table } 2 \text { (Nitrogen) } \\
\text { Table } 3 \text { (Beryllium) }\end{array}$ \\
\hline$\tau$ & $\begin{array}{l}\text { Transition time in the gas } \\
\text { attenuator }\end{array}$ & Eq. 3.11 \\
\hline
\end{tabular}




\section{Introduction}

The Linac Coherent Light Source [1] that has just started its operation at Stanford, will provide new unique platform for a broad range of experiments using extremely intense, coherent pulses of X-rays. The design pulse energy for the coherent radiation is approximately $2 \mathrm{~mJ}$. The pulses will follow at the repetition rate of $f=120 \mathrm{~Hz}$ thereby allowing for collecting good statistics. The energy range of coherent X-rays in LCLS can be varied in a controlled fashion between approximately $826 \mathrm{eV}$ and $8260 \mathrm{eV}$.

In a variety of experiments, it may be desirable to make a scan of the observed effects vs the pulse intensity. To change it with respect to the maximum value of $2 \mathrm{~mJ}$ without re-tuning the accelerator/undulator part, an X-ray beam attenuator will be used. It consists of a combination of the gas attenuator (which is the main subject of our report) and the solid attenuator. Those can be used separately or simultaneously, providing the attenuation up to a design factor of $10^{4}$.

A general schematic of the gas attenuator is shown in Fig. 1.1. The working gas enters the system through the inlet in the middle of the attenuator and fills the volume of the gas attenuator. As the line of site cannot be blocked, the attenuator volume is directly connected to the accelerator volume (a windowless system), and one needs to use a differential pumping system to separate the gas attenuator (with a relatively high gas pressure in it) and the beamline (where high vacuum has to be maintained).

Gas attenuator allows one to vary in a continuous manner the beam intensity downstream the beamline by simply varying the calibrated inflow of gas. Its other obvious advantage is that it can be controlled without any mechanically moving parts in the beamline.

The attenuation is caused by the photoabsorption and, to a lesser degree, by the Compton scattering in the working gas. In the past, several working gases have been considered [2-4], in particular, xenon, argon, and nitrogen. Eventually, the nitrogen was chosen for the LCLS system. It has several advantages: It does not have any K-transitions in the energy range of importance for LCLS, and thereby has a smooth dependence of the absorption coefficient vs. energy. It is inexpensive and does not need to be recirculated. Being lighter than air, it has some safety advantages over more heavy gases. Its relative disadvantage is a small photoabsorption cross-section, especially at the higher end of the energy range.

The pressure in the attenuation cell is limited by the capabilities of the differential pumping system and cannot exceed 10 torr in the present design. The length of the attenuation cell is approximately $420 \mathrm{~cm}$ and could not be significantly increased due to the space limitations. These two constraints determine the maximum achievable line density of nitrogen and, thereby, the maximum achievable attenuation. With the aforementioned limitations on the length and the pressure, the gas attenuator alone can provide the attenuation coefficient $10^{4}$ only at the energies below roughly $1500 \mathrm{eV}$ (see Sec. 2 for more detail), whereas at the energy of, say, $4 \mathrm{keV}$ the maximum attenuation is only 1.4. On the other hand, the LCLS design specifications require that the attenuation be as high as $10^{4}$ over the whole X-ray energy range from 826 to $8260 \mathrm{eV}$. 


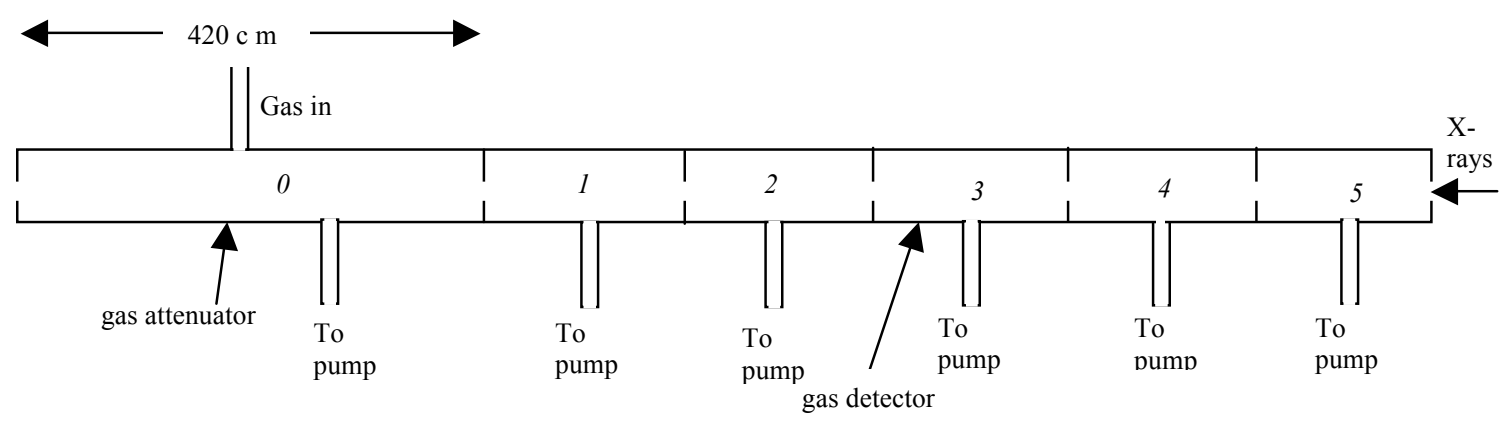

Fig. 1.1 Schematic of the gas attenuator/gas detector system (not to scale). The length of the attenuator is $420 \mathrm{~cm}$, the lengths of the subsequent cells are $31 \mathrm{~cm}$ (cell 1), $41 \mathrm{~cm}$ (cell 2), $48 \mathrm{~cm}$ (cell 3, gas detector), $39 \mathrm{~cm}$ (cell 4) and $39 \mathrm{~cm}$ (cell 5). Only the upstream part of the differential pumping system is shown. There is an identical set of cells (including one more gas detector) attached to the left aperture of the gas attenuator. A more realistic rendition of the whole system is shown in Fig. 1.2.

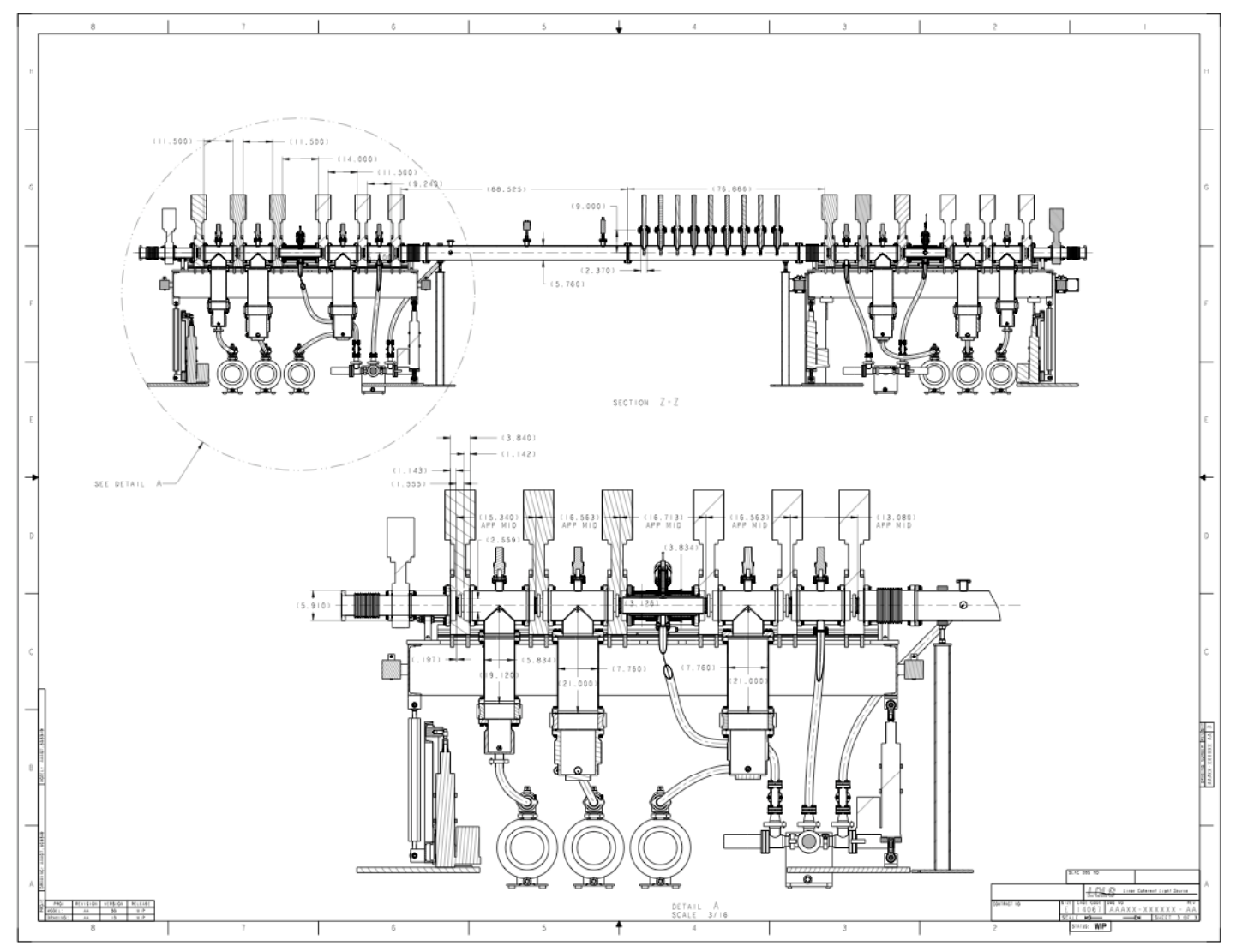

Fig. 1.2 Detailed drawing of the gas attenuator in combination with the solid attenuator. In the position shown in the figure, all the beryllium slabs are "in." The X-ray beam enters the system from the right. 
In order to provide required attenuation at higher energies, a solid attenuator is combined (geometrically and functionally) with the gas attenuator. Beryllium foils and slabs can be inserted into the beam line, as shown in Fig. 1.2. Their various combinations allow one to make attenuation as high as $10^{4}$ for all the energies in the aforementioned range. If needed, the solid attenuator can be used in combination with the gas attenuator, thus allowing for a smooth variation of the attenuation over the whole energy range. As the solid attenuator is an inseparable part of the whole attenuation system, we briefly discuss physics issues affecting its performance as well.

This report does not discuss the long process of trial and error that eventually led to the chosen set of parameters. We focus on the pressure range between zero and 10 torr - the maximum value compatible with the present design of the vacuum system. Specific constraints imposed by the vacuum requirements for the adjacent section of the beamline (in particular, the maximum size of the apertures) have been considered in great detail in earlier reports [3-7] and we do not dwell on them any more.

Although very simple in principle, the performance of the gas attenuator involves a broad variety of processes with disparate temporal and spatial scales, from the photoionization during the passage of the X-ray pulse (hundreds of femtoseconds), through subsequent secondary ionizations and heating of the gas (picoseconds to tens of milliseconds), to very slow processes of surface modification by active products of X-ray interactions with nitrogen (days and months). The processes discussed below have fourfold implications for the operation of the attenuator: first, they may affect the accuracy to which the attenuation coefficient can be set; second, they may cause distortions of the Xray wave-front; third, they may affect the aging of the attenuator components; finally, some of them may serve as a basis for new techniques of the X-ray beam characterization (like imaging or total energy measurements) in future facilities.

As the parameter domain for the system is very broad, most of the figures have to use logarithmic scales and can provide an accuracy of $15-20 \%$ at best. In order to provide more quantitative reference numbers we duplicate some of the figures by tables.

\section{Beam attenuation}

\subsection{Evaluating the attenuation}

The attenuation coefficient $A$ is defined as a ratio of the pulse energy before and after the attenuator ( $W_{\text {in }}$ and $W_{\text {out }}$, respectively):

$$
A=\frac{W_{\text {in }}}{W_{\text {out }}}
$$

We use data from Ref. [8] to evaluate attenuation for the temperature of $25 \mathrm{C}$. The attenuation coefficient can be expressed as

$$
A=\exp \left[0.00125\left(\mathrm{~g} / \mathrm{cm}^{3}\right) \Sigma\left(\mathrm{cm}^{2} / \mathrm{g}\right) \frac{p(\text { torr }) L(\mathrm{~cm})}{760}\right] \text {, }
$$

where the first coefficient in the exponent is the nitrogen gas density at the pressure of 760 torr and the temperature of $25 \mathrm{C}, \Sigma$ is the sum of photo-absorption and inelastic scattering cross-sections from Ref. [8], $p$ is the pressure in the gas attenuator, and $L$ is its 


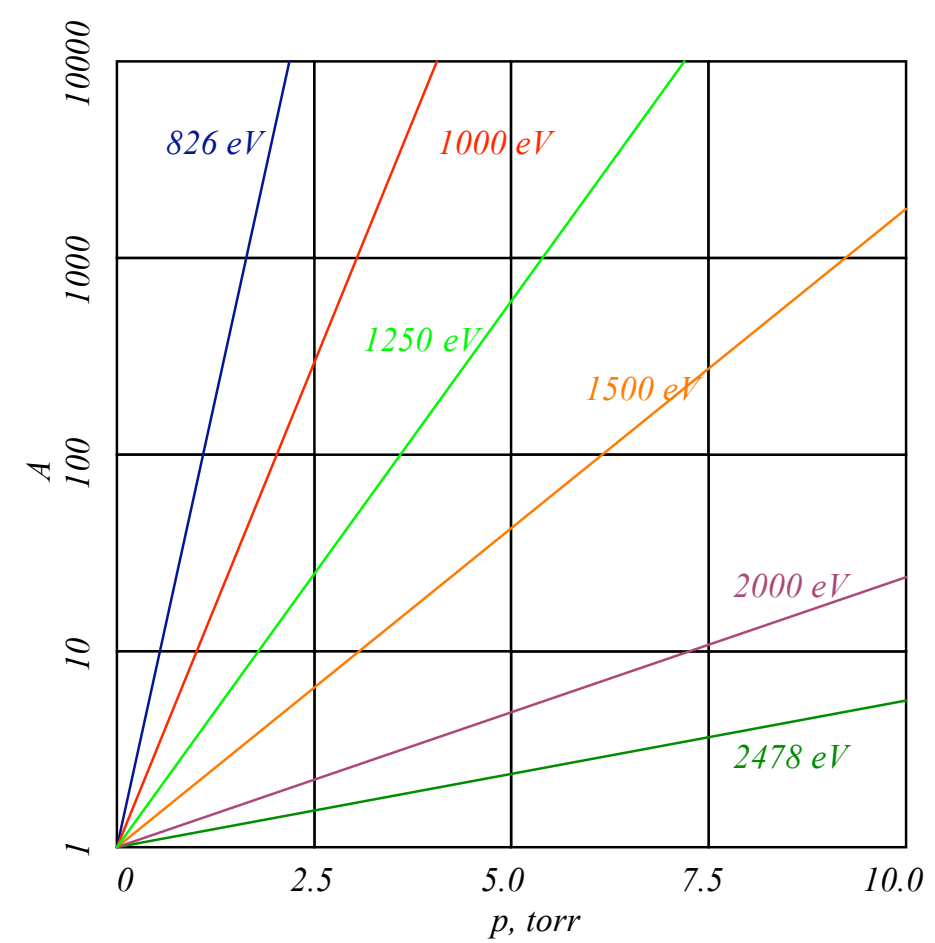

Fig. 2.1 Attenuation coefficient at lower X-ray energies. Note that the vertical scale is logarithmic. The attenuator length is assumed to be $420 \mathrm{~cm}$.

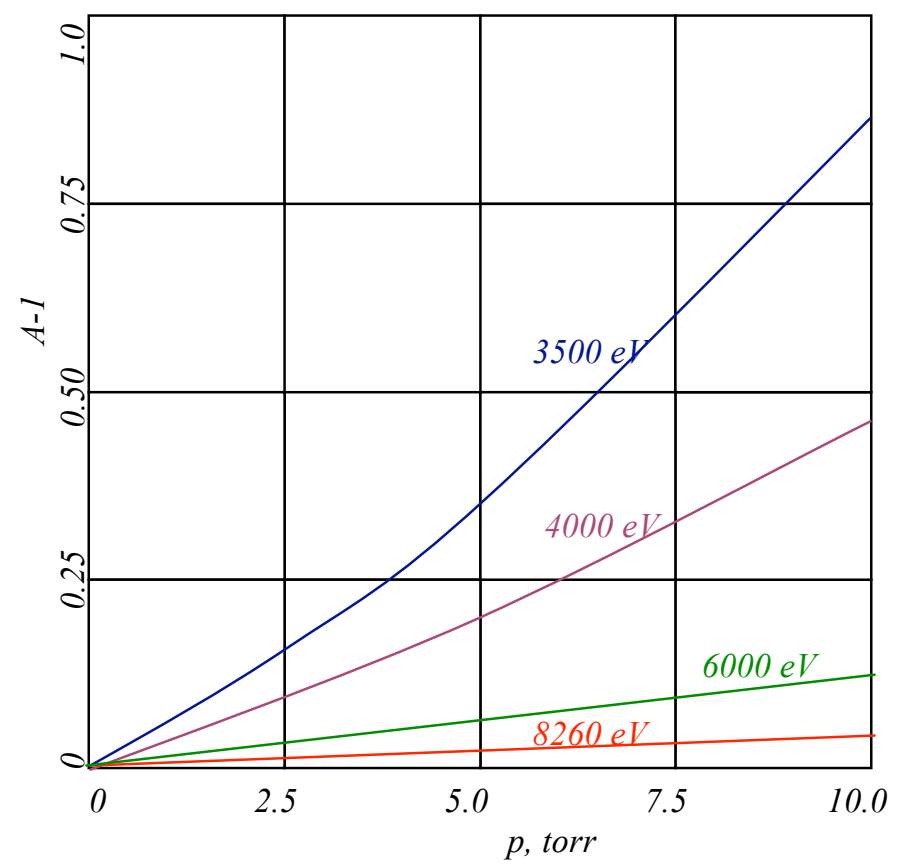

Fig. 2.2 Attenuation coefficient at higher X-ray energies. Note that the vertical scale is linear and the quantity shown is $A-1$. The attenuator length is assumed to be $420 \mathrm{~cm}$. 
length between the entrance and exit apertures. Throughout this section we assume that $L=420 \mathrm{~cm}$. With that assumption, Eq. (2.2) can be also presented as

$\log A=0.0003 p($ torr $) \Sigma\left(\mathrm{cm}^{2} / \mathrm{g}\right)$

where $\log$ means a decimal logarithm. This relation is illustrated by Fig. 2.1, for the $\mathrm{X}$ ray energies between the lower end of the operation domain, $826 \mathrm{eV}$, and the third harmonic, $2478 \mathrm{eV}$. One sees that for the photon energies below, roughly, $1500 \mathrm{eV}$, the gas attenuator alone can provide the maximum attenuation of $10^{4}$ required by the LCLS specifications. At the energy $\sim 2.5 \mathrm{keV}$ it can provide significant attenuation, by a factor of a few; reaching higher attenuations requires using the solid attenuator in combination with the gas attenuator.

Table 1 Absorption cross-section and e-folding attenuation length for nitrogen

\begin{tabular}{|l|l|l|}
\hline X-ray energy $E_{x}, \mathrm{keV}$ & $\begin{array}{l}\text { Absorption cross-section } \\
\Sigma, \mathrm{cm}^{2} / \mathrm{g}\end{array}$ & $\begin{array}{l}\text { e-folding absorption length, } \\
l_{\text {abs}, ~} \mathrm{~cm}, \text { for } p=10 \text { torr* and } T=25 \mathrm{C}\end{array}$ \\
\hline 0.826 & 5498 & 11.05 \\
\hline 1.00 & 3306 & 18.3 \\
\hline 1.50 & 1079 & 56.3 \\
\hline 2.00 & 474 & 128 \\
\hline 3.00 & 144 & 422 \\
\hline 4.00 & 60 & 1013 \\
\hline 6.00 & 17.3 & 3514 \\
\hline 8.00 & 7.1 & 8563 \\
\hline 8.26 & 6.4 & 9500 \\
\hline
\end{tabular}

* For other pressures, the e-folding length can be found by introducing a multiplier 10/p(torr).

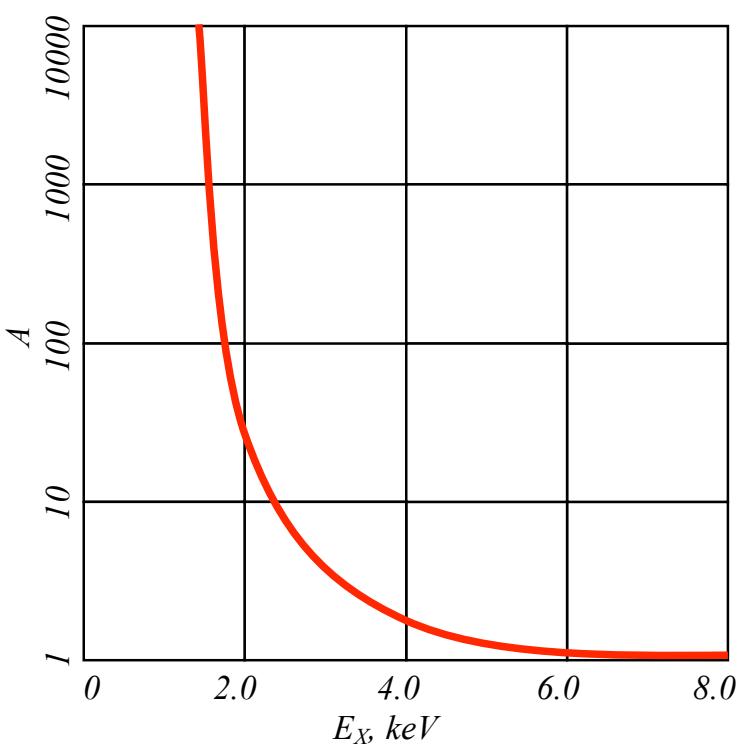

Fig. 2.3 Attenuation coefficient vs X-ray energy for the pressure of 10 torr.

At the energies exceeding $2.5 \mathrm{keV}$ and up, the gas attenuator can be used for fine tuning of the attenuation produced in discrete steps by the solid attenuator. The possible 
degree of the fine tuning falls below $5 \%$ at the energy of $8260 \mathrm{eV}$. This is illustrated in Fig. 2.2, where the parameter $A-1$ is plotted vs. the pressure $p$ for several energies beyond $2.5 \mathrm{keV}$. If the operational temperature is different from $T=25 \mathrm{C}$, then there should be a correction factor $298.15 /(273.15+T)$ introduced to the exponent of Eq. (2.2) and to the r.h.s. of Eq. (2.3). The attenuation coefficient vs. the X-ray energy is plotted in Fig. 2.3 for $p=10$ torr.

\subsection{Errors in the attenuation coefficient}

Equation (2.2) can obviously be presented as $A=\exp \kappa$, where $\kappa$ is the exponent in Eq. (2.2). An error $\delta \kappa$ in the exponent generates an error $\delta A$ in the attenuation coefficient, $\frac{\delta A}{A}=\frac{\delta \kappa}{\kappa} \ln A$.

For $\delta \kappa / \kappa$ one has:

$$
\frac{\delta \kappa}{\kappa}=\frac{\delta p}{p}-\frac{\delta T}{T}+\frac{\delta \Sigma}{\Sigma}
$$

The second term appears here due to the assumption made in Eq. (2.2) that the density of the gas corresponds to the temperature of $25 \mathrm{C}$.

There may be systematic and statistical errors in measurements of the pressure and the temperature. There may be also a systematic error associated with the imperfect knowledge of the cross-section. Another source of error may creep in due to statistical variations of the X-ray energy, this leading to statistical variation of the cross-section. The effect of errors is maximum at maximum attenuations, as manifested by the presence of the coefficient $\ln A$ in Eq. (2.4).

In addition to these obvious sources of error, there is also additional statistical source associated with the possible presence of the turbulence in the gas flow between the attenuator and the adjacent stages of the differential pumping system. We discuss this effect in Sec. 3 and find that it is generally small.

A more subtle effect is associated with the head-to-tail variation in the ionization degree of the gas through which the beam propagates: The front part of the beam propagates through a virgin gas, whereas the tail "sees" the gas that has already been partially ionized and whose absorption coefficient has somewhat changed. This effect is discussed in Sec. 3.6 and is found to be insignificant.

Finally, at highest intensities of the beam, there may occur some average (over many pulses) heating of the gas near the axis of the attenuator. The corresponding temperature increase near the axis will change the attenuation compared to the result expected from the temperature measurement at the wall. This effect is discussed in Sec. 3.3 . 


\section{Gas flow in the attenuator and the differential pumping system}

\subsection{Gas flow through the apertures}

Some important gas-kinetic parameters for the nitrogen are listed in Table 2.

The mean free path for the nitrogen molecules can be evaluated as $\lambda=1 / n \sigma$, where $n$ is the particle density, and $\sigma$ is the collision cross-section. [Note that we do not include the factor $2^{-1 / 2}$ in definition of the free path.] For the particle density $n$ one has:

$n\left(\mathrm{~cm}^{-3}\right)=3.51 \times 10^{16} \mathrm{p}($ torr $)$,

so that the mean-free-path $\lambda$ is

$\lambda(\mathrm{cm}) \approx \frac{7.5 \times 10^{-3}}{p(\text { torr })}$.

Compare $\lambda$ with the radius $a$ of the apertures connecting gas attenuator with the adjacent cells. The plot of the ratio $a / \lambda$ is shown in Fig. 3.1. One sees that in the wide range of pressures this ratio is much greater than 1 , meaning that the flow is in most cases collisional, hydrodynamic flow (as opposed to the Knudsen flow). This conclusion holds with an even larger margin with respect to the flow along the gas attenuator itself: the radius $b$ of the attenuator chamber is approximately $7 \mathrm{~cm}$, so that $b / \lambda$ is 35 times larger than $a / \lambda$. The sketch of the streamlines in the gas attenuator is shown in Fig. 3.2.

Table 2. Some parameters of the nitrogen gas $[15,16]$.

\begin{tabular}{|l|l|l|l|l|l|l|l|}
\hline $\begin{array}{l}\text { Molecular } \\
\text { diameter, } d\end{array}$ & $\begin{array}{l}\text { Elastic } \\
\text { cross- } \\
\text { section, } \\
\sigma=\pi d^{2}\end{array}$ & $\begin{array}{l}\text { Viscosity } \eta \\
\text { at } T=20 \mathrm{C} \\
\text { and } p=760 \\
\text { torr }\end{array}$ & $\begin{array}{l}\text { Kinematic } \\
\text { viscosity } v \\
\text { at } T=20 \mathrm{C} \\
\text { and } p=760 \\
\text { torr }\end{array}$ & $\begin{array}{l}\text { Thermal } \\
\text { diffusivity } \chi \\
\text { at } T=20 \mathrm{C} \\
\text { and } p=760 \\
\text { torr }\end{array}$ & $\begin{array}{l}\text { Density, } \rho \\
\text { at } T=25 \mathrm{C} \\
\text { and } p=760 \\
\text { torr }\end{array}$ & $\begin{array}{l}\text { Adiab. } \\
\text { index } \gamma\end{array}$ & $\begin{array}{l}\text { Sound } \\
\text { speed } s_{0} \text { at } \\
\mathrm{T}=25 \mathrm{C}\end{array}$ \\
\hline $3.5 \times 10^{-8} \mathrm{~cm}$ & $\begin{array}{l}3.8 \times 10^{-15} \\
\mathrm{~cm}^{2}\end{array}$ & $\begin{array}{l}1.775 \times 10^{-4} \\
\mathrm{~g} / \mathrm{cm} \cdot \mathrm{s}\end{array}$ & $0.14 \mathrm{~cm}^{2} / \mathrm{s}$ & $0.2 \mathrm{~cm}^{2} / \mathrm{s}$ & $\begin{array}{l}0.00125 \\
\mathrm{~g} / \mathrm{cm}^{3}\end{array}$ & 1.4 & $\begin{array}{l}3.5 \times 10^{4} \\
\mathrm{~cm} / \mathrm{s}\end{array}$ \\
\hline
\end{tabular}

The flow from the gas attenuator to the neighboring cell is a transonic flow, with the local flow speed reaching the local sonic speed at the center of the aperture. This speed, which we denote as $s^{*}$, can be expressed in terms of the sound speed $s_{0}$ in the midplane of the attenuator (see, e.g., Ref. [9]):

$s^{*}=s_{0} \sqrt{\frac{2}{\gamma+1}}$.

The particle density $n *$ in the transition point is

$n^{*}=n_{0}\left(\frac{2}{\gamma+1}\right)^{\frac{1}{\gamma-1}}$.

Note that the gas temperature in the transition point is significantly lower than in the middle of the attenuator, 
$T^{*}=\frac{2 T_{0}}{\gamma+1} \approx 248 K$

or $-25 \mathrm{C}$. This lower temperature region is, however fully decoupled from the main volume of the attenuator, due to a rapid flow in the aperture area. It does not affect the average temperature $T_{0}$ in any significant way.

After having reached the critical sonic point, the gas continues to expand and cool down in the first cell of the differential pumping system. It eventually recompresses via shock transition at a distance of $\sim 1 \mathrm{~cm}$ from the orifice and mixes up with a slowly flowing gas in the first cell.

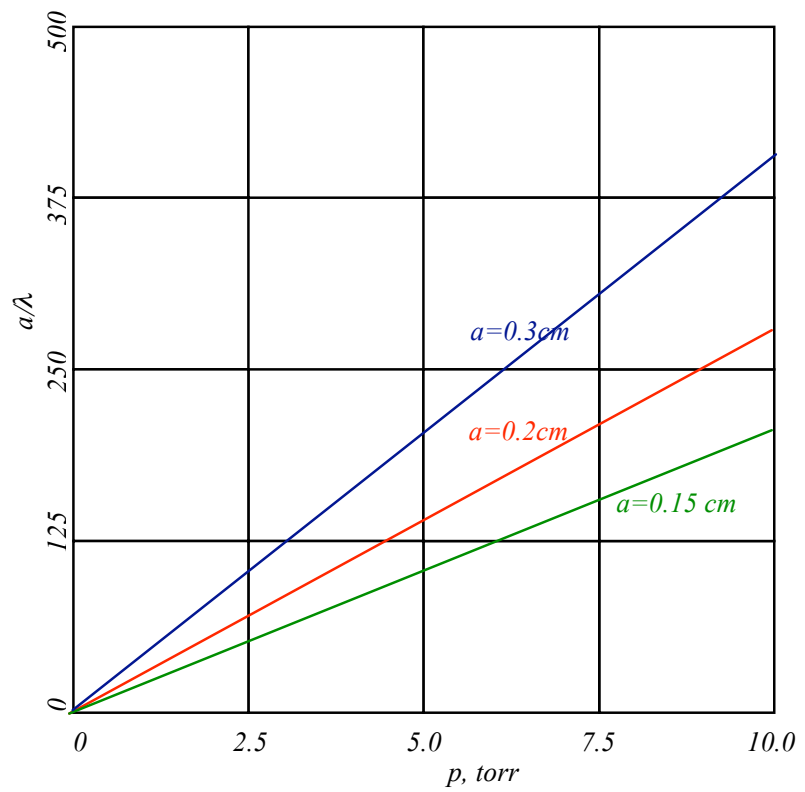

Fig. 3.1 The ratio of the aperture radius a to the mean free path vs the gas pressure. The flow remains collisional even at the pressure of 0.1 torr.

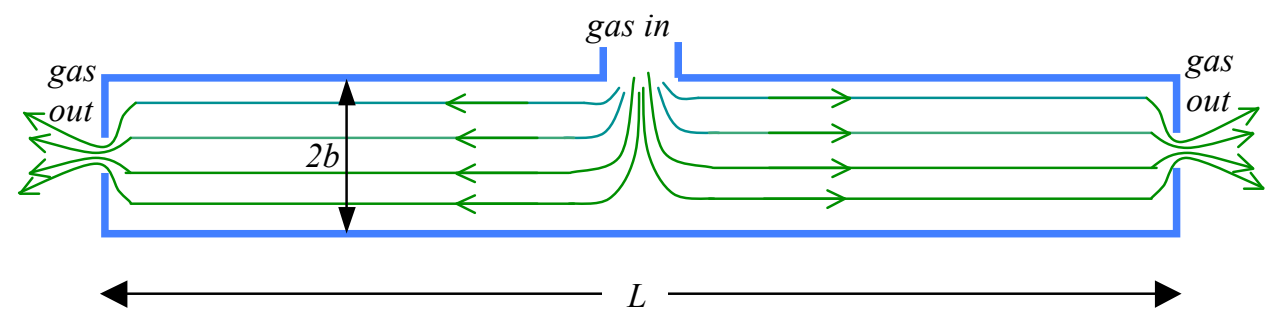

Fig. 3.2 Schematic of streamlines (green) in the gas attenuator. The Reynolds number for this flow is well below the critical one. 
The sketch of the density distribution in the transition region is shown in Fig. 3.3. The post-shock density is approximately equal to the density $n_{1}$ in the cell 1 . The latter is determined by the gas inflow from the attenuator and the pump speed. For the presently accepted pump system, it is approximately 0.1 of the density in the attenuator (K. Kishiyama). The pre-shock density $n^{* *}$ (Fig. 3.3) is related to the post-shock density $n_{l}$ by a strong-shock condition,

$$
n * *=\frac{\gamma-1}{\gamma+1} n_{1}
$$

For $\gamma=7 / 5$ and the density $n_{1} \sim 0.1 n_{0}$, this pre-shock density is quite low, $\sim n_{0} / 60$. In other words, a significant expansion of the supersonic flow occurs before it gets recompressed via the shock transition. This leads to a cooling of the gas to quite a low temperature of $T^{* *}=T_{0}\left[\left(n_{1} / n_{0}\right)(\gamma-1) /(\gamma+1)\right]^{\gamma-1} \approx 58 K$. In the shock transition the gas is reheated to approximately the room temperature.

All these effects are separated from the main volume of the attenuation cell by the strong supersonic outflow through a small orifice and do not have any significant influence on the processes in the attenuator. One could be concerned with a process of the cluster formation in the cold, rapidly expanding nitrogen, but the density here is relatively low and the transit time between the nozzle and the shock is too short for the cluster of any significant size to form. [Large clusters, if they intersected the beam, might lead to random fluctuations in the attenuation.]

Equations (3.3a) and (3.3b) allow one to evaluate the gas throughput. It is (in particles per second)

$$
\dot{N}=2 \pi a^{2} n^{*} s^{*}=2 \pi a^{2} n_{0} s_{0}\left(\frac{2}{\gamma+1}\right)^{\frac{\gamma+1}{\gamma-1}} \approx 2.1 a^{2} n_{0} s_{0}
$$

We account here for the losses through both ends. Multiplying this number by the molecular weight, using Eqs. (3.1) and (3.6) and substituting $s_{0}$ for the temperature of 298 $\mathrm{K}$ from Table 1, one finds a mass throughput:

$$
\dot{M}(g / s)=4.8 \cdot 10^{-3} p(\text { torr }) \text {. }
$$

One can also express this result in terms of torr $1 / \mathrm{s}$

$\dot{U}($ torr $\cdot l / s)=0.29 p($ torr $)$.

In Eqs. (3.7) and (3.8) we assumed $a=0.2 \mathrm{~cm}$ and $T=298 \mathrm{~K}$.

\subsection{Gas flow inside the gas attenuator}

The flow inside the gas attenuator is very slow, except for the immediate vicinity of the gas inlet area. Axial velocity in the midplane is near zero, and is directed away from the mid-plane in the left and right halves of the attenuator. The overall sketch of the streamlines is shown in Fig. 3.2.

To evaluate axial velocity $\mathrm{v}$ halfway between the inlet and the end of the attenuator, one can use the equation

$$
\pi b^{2} n_{0} \mathrm{v}=\pi a^{2} n_{0} s_{0}\left(\frac{2}{\gamma+1}\right)^{\frac{\gamma+1}{\gamma-1}}
$$




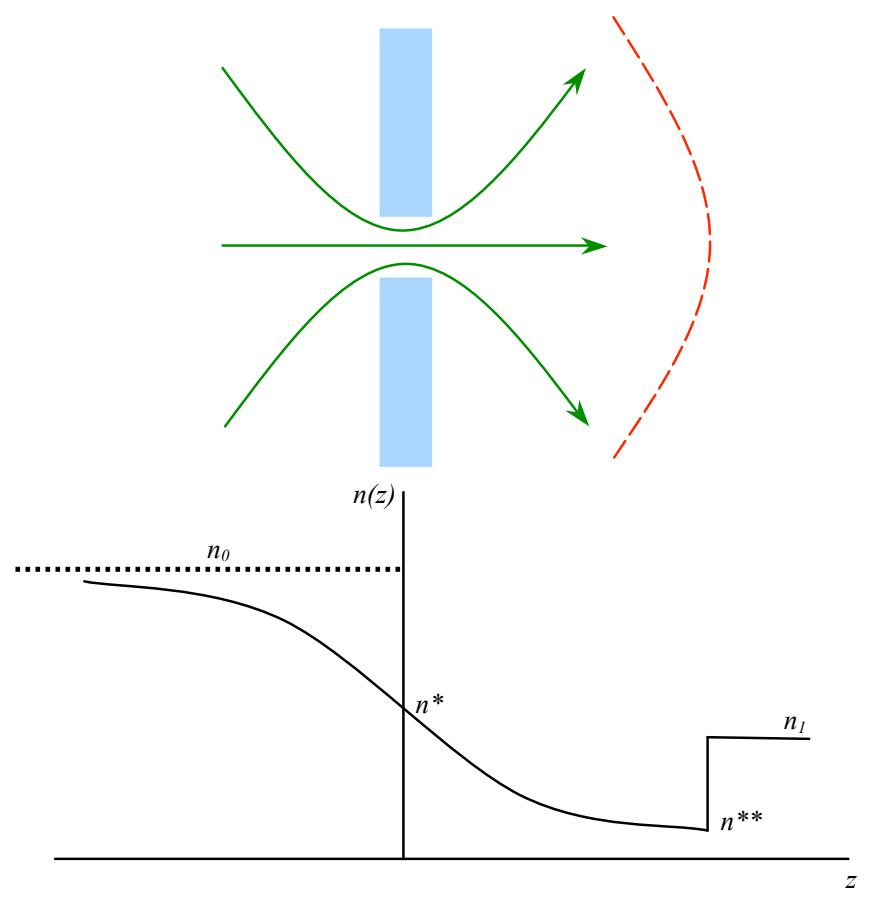

Fig. 3.3 Transonic flow through the beryllium-carbide aperture (blue). Streamlines are shown in green, red dashed line shows the position of the shock. The lower panel shows a sketch of the density distribution along the axis (not to scale). The density first drops significantly in the supersonic expanding flow in the adjacent (to the gas attenuator) cell and then increases via the shock transition.

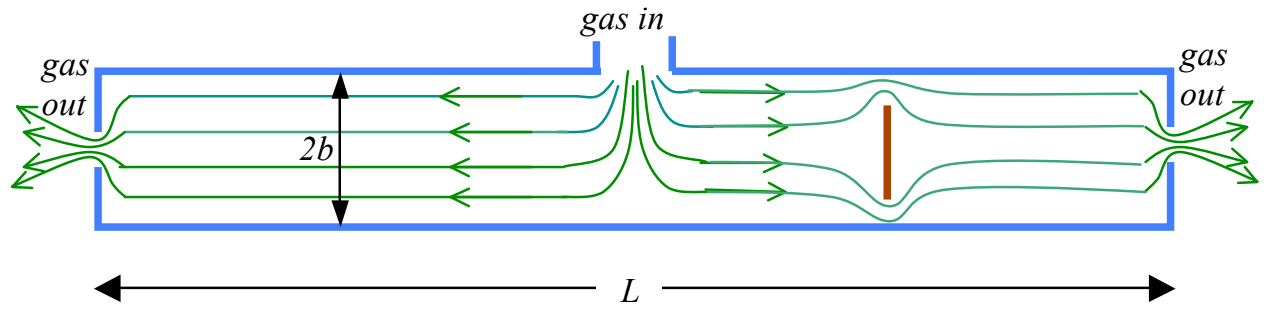

Fig. 3.4 Schematic of streamlines in the gas attenuator with one of the attenuation blocks in the "in" position (thick brown line). The support structure holding the block in place is not shown. Although the flow velocity in the gaps between the block and the walls increases, it is still too low to cause any noticeable pressure difference between the end flanges. 
For $a=0.2 \mathrm{~cm}$ and $b=7 \mathrm{~cm}$, one finds that the flow velocity is deeply subsonic, $\mathrm{v} / s_{0} \approx 2.7 \times 10^{-4}$. This means that the pressure variations associated with this flow will be negligibly small: according to Bernoulli equation, they will be of the order of $\Delta p_{d y n} \equiv \rho \mathrm{v}^{2} \sim 10^{-7} p$. The presence of the obstructions to the flow produced by clamps and foils of a solid attenuator (when they are deployed) reduce the flow cross-section by a factor of $\sim 2$ and lead to some increase in the flow velocity around the clamps, but the associated pressure variation is still negligibly small.

One more contribution to the pressure variation along the tube may be caused by the viscous friction of the flowing gas against the walls. This part of the pressure variation can be evaluated as $\Delta p_{\text {visc }} \equiv \eta v L / b^{2}$. Substituting $\eta$ from Table 2 and taking $\mathrm{v} \sim 2.7 \times 10^{-4} s_{0} \sim 10 \mathrm{~cm} / \mathrm{s}$, one finds: $\Delta p_{\text {visc }} \sim 6 \times 10^{-6}$ torr, which is negligible. The presence of the obstructions somewhat increases the viscous friction in the corresponding part of the attenuator, but even 10-fold increase does not give rise to any appreciable pressure variation.

Therefore, we conclude that the pressure in the gas attenuator is uniform to a high degree, and the gas outflow from both ends is the same, irrespectively to whether the solid attenuator is deployed or not.

An important parameter is the time constant for the change of the gas density in the case where one needs to switch from one to another attenuation level. The equation describing the time dependence of the gas inventory in the attenuation cell is Eq. (3.6). Substituting into it $N=n_{0} \mathrm{~V}$, where $\mathrm{V}$ is the attenuator volume, and adding a source term $\dot{N}_{\text {source }}$ (controlled gas flow through the inlet), one finds:

$\dot{N}=\dot{N}_{\text {source }}-\frac{N}{\tau}, \tau=\frac{V}{2.1 a^{2} s_{0}}$.

The time constant $\tau$ is large, a few tens of seconds. Assuming that $V=\pi b^{2} L$, and substituting $b=7 \mathrm{~cm}, L=420 \mathrm{~cm}, s_{0}=3.5 \times 10^{4} \mathrm{~cm} / \mathrm{s}$, we find $\tau \approx 22 \mathrm{~s}$. One has to wait for (23) $\tau$ before the attenuator reaches the new steady state after the change of the gas input.

If the gas input experiences fast (compared to $\tau$ ) change by some amount $\Delta \dot{N}_{\text {source }}$, the gas inventory varies as

$\Delta N=\tau \Delta \dot{N}_{\text {source }}\left(1-e^{-t / \tau}\right)$,

where $\tau$ is introduced in Eq. (3.10). This circumstance can be used for the experimental cross-check of the validity of the whole analysis, by comparing the pressure evolution measured by the baratron gauges with the dependence predicted by Eq. (3.11).

\subsection{Gas heating by X-ray pulses}

Initial photoelectrons (plus Auger electrons) create secondary ionizations and excitations and gradually lose their energy. They eventually slow down to the energies below electronic excitations and start exchanging their energy with vibrational, rotational and translational degrees of freedom, thereby leading to the heating of the gas. We characterize a fraction of the absorbed energy that is dissipated into the thermal energy of the gas (not radiated away) by the dimensionless parameter $\eta<1$. 
The heating is strongest near the entrance to the attenuator, where the X-ray pulse energy is maximum (not yet attenuated). We, therefore, concentrate on the processes near the entrance. The energy $q$ deposited per unit length here is equal to $q=\rho_{0} \Sigma W$,

where $\rho_{0}$ is the mass density of the gas in the attenuator, the parameter $\Sigma$ is a crosssection from Ref. [8] introduced after Eq. (2.2), and $W$ is the initial (not attenuated) pulse energy. For the gas with the initial temperature of $25 \mathrm{C}$, one can present the result (3.12) also as $q(\mathrm{~J} / \mathrm{cm})=1.6 \times 10^{-9} p_{0}($ torr $) \Sigma\left(\mathrm{cm}^{2} / \mathrm{g}\right) W(\mathrm{~mJ})$.

The initial energy deposition by the X-ray pulse occurs essentially instantaneously. The further spatio-temporal evolution depends on the processes of the energy exchange between the electrons and the gas, gas-dynamic expansion of the heated region, and radial spreading of the heat by the thermal conductivity.

An important role at not-too-small gas pressures is played by the electrostatic confinement of the primary photoelectrons [10]: they cannot expand radially, because they are held by the space charge of the positive ions, whose expansion is slow due to their large mass. So, for most conditions of relevance to the gas attenuator operational domain, the primary photoelectrons deposit their energy within the aperture of the X-ray beam. All this happens within the time not longer than a few tens of nanoseconds (see Fig. 5 in Ref. [10]); therefore, the acoustic expansion of the heated volume and the thermal conduction can be neglected during this phase (see below for the corresponding estimates).

The condition for the strong electrostatic confinement of the primary photoelectrons can be presented as (see Eq. (8) in Ref. [10]):

$p($ torr $)>p_{\text {el-stat }} \equiv 160 \frac{E_{X}(\mathrm{keV})\left[E_{X}(\mathrm{keV})-0.4\right]}{\Sigma\left(\mathrm{cm}^{2} / \mathrm{g}\right) W(\mathrm{~mJ})}$

[Note a typo in Eq. (8) of Ref. [10], where the factor $W$ is missing in the denominator.] This result is illustrated by Fig. 3.5. One sees that, at the energies below $2.5 \mathrm{keV}$ electrostatic confinement is important even for the pulses with the energy contents tens of times less than the nominal energy content of $2 \mathrm{~mJ}$.

The maximum energy deposition occurs on the beam axis. We assume the Gaussian radial distribution of the beam fluence, $F=F_{0} \exp \left(-2 r^{2} / w^{2}\right)$,

where $w$ is a parameter characterizing the beam width. The on-axis fluence $F_{0}$ is related to the total pulse energy $W$ by:

$F_{0}=2 \mathrm{~W} / \pi w^{2}$.

The thermal energy $Q$ deposited per unit volume at the beam axis is, obviously,

$Q=\eta \rho_{0} \Sigma F_{0}=\eta \frac{2 \rho_{0} \Sigma W}{\pi w^{2}}$,

where $\eta$ is the aforementioned "efficiency" of the energy transfer from the electrons to thermal energy of the gas. In "practical" units,

$Q\left(J / \mathrm{cm}^{3}\right)=0.052 \frac{\eta p_{0}(\text { torr }) \Sigma\left(\mathrm{cm}^{2} / \mathrm{g}\right) W(\mathrm{~mJ})}{[w(\mu \mathrm{m})]^{2}}$. 


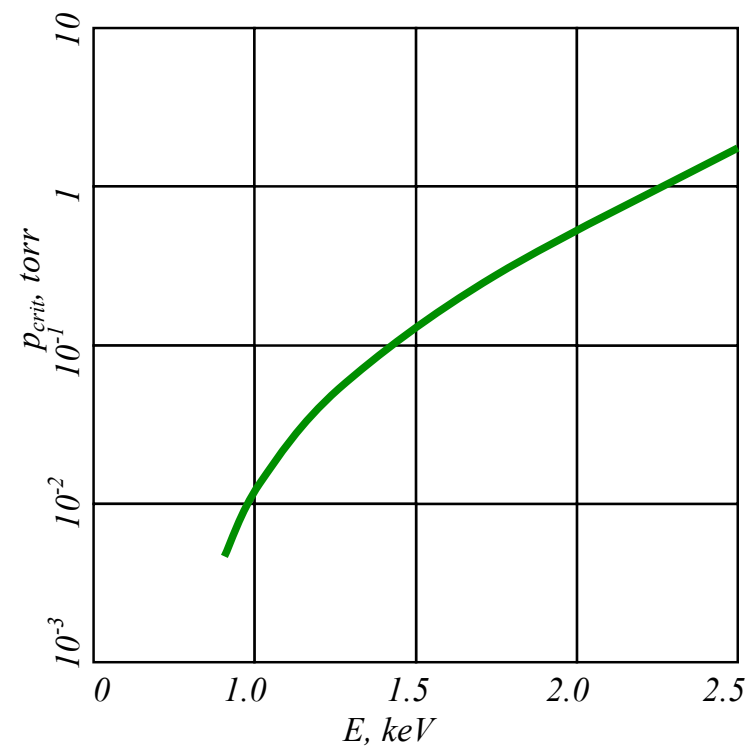

Fig. 3.5 Critical pressure corresponding to the onset of the "electrostatic confinement" of the primary photoelectrons by the space-charge of the ions.

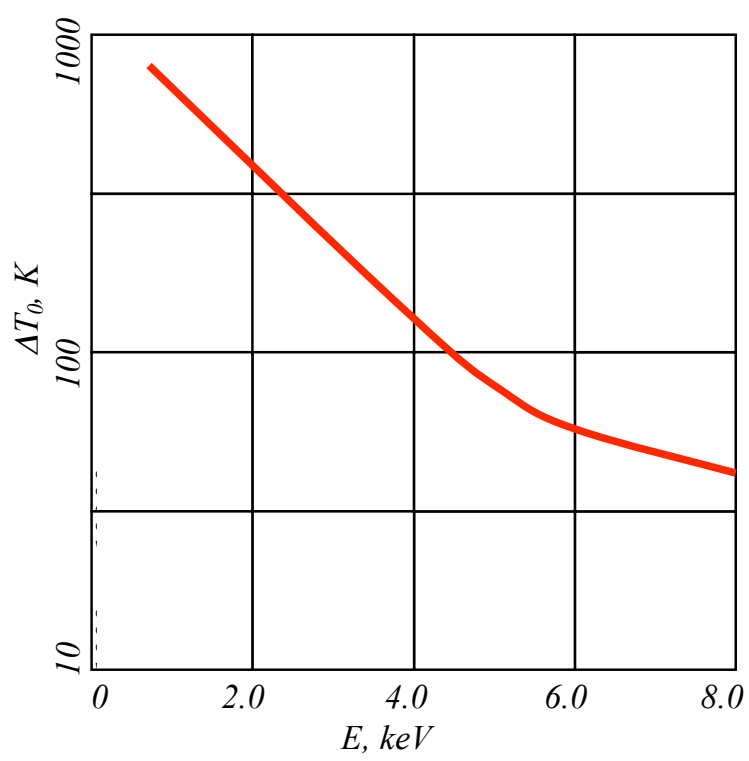

Fig. 3.6 Isochoric on-axis temperature increase (no gas expansion or thermal conduction accounted for); pulse energy $W=2 \mathrm{~mJ}$, efficiency $\eta=0.5$. 
Assuming that the heating occurs without radial expansion of the heated zone, one can find the maximum possible temperature increase on the beam axis just by dividing $Q$ by $c_{\mathrm{V}}$, the isochoric specific heat per unit volume. Taking the value of $c_{\mathrm{V}}$ for the ideal diatomic gas, one finds the initial temperature increase:

$\Delta T_{0}(K)=1.34 \times 10^{5} \frac{\eta \Sigma\left(\mathrm{g} / \mathrm{cm}^{2}\right) W(\mathrm{~mJ})}{[w(\mu \mathrm{m})]^{2}}$.

Note that it does not depend on the gas density (gas pressure). The plot of $\Delta T_{0}$ vs the photon energy is presented in Fig. 3.6, for the pulse energy $W=2 \mathrm{~mJ}$ and $\eta=0.5$.

At the lower end of the photon energies, $\Delta T_{0}$ is very high, several hundreds $\mathrm{K}$. One has, however, to remember that $\Delta T_{0}$ is the maximum possible temperature increase, which has to be used just as a reference point, with the understanding that the real temperature increase may be significantly lower due to the radial broadening of the heated area.

As we have already mentioned, the initial beam energy deposition occurs virtually instantaneously, but the further spatio-temporal evolution of the deposited energy involves a series of slower processes depending on the space-charge effects, rate of the energy exchange between the electrons and molecules, the gas-dynamics and thermal conduction of the heated gas. These processes may lead to a significant decrease of the temperature (compared to the estimate (3.19)) prior to the arrival of the next pulse.

At lower pressures, the broadening of the heated channel may start before the electrons exchange energy with the neutrals. The duration of this process is determined by the electrons with the energies in the range of $1-3 \mathrm{eV}$ [7], which cannot produce ionizations or electronic excitations. The energy exchange time between such electrons and the gas can be evaluated as (Ref. [10]):

$$
\tau_{\text {e-gas }}(s) \sim \frac{2 \times 10^{-5}}{p(\text { torr })}
$$

For the pressures near the maximum allowable one (10 torr), this time is short, of order of a few microseconds, shorter than acoustic crossing time over $\sim 1 \mathrm{~mm}$ radius of the initially heated cylinder. So, at high pressures, the maximum temperature can indeed reach the value determined by Eq. (3.19). However, for the lower pressures, the energy exchange will be slower and will be accompanied by the expansion of the heated zone driven by the higher pressure inside it. In either case of fast or slow energy exchange between the electrons and the gas, at later times the deposited energy is spread over the area larger than the beam radius. After a few reverberations, the heated channel reaches the pressure equilibrium with the external medium.

The heat conduction leads to a further broadening of the heated zone and reduction of the temperature. By the time of the arrival of the next pulse, the radius of the channel will be of order of

$r_{\text {therm }} \sim \sqrt{2 \chi t}$,

with $\chi$ being the thermal diffusivity (Table 2 ) and $t=1 / 120 \mathrm{~s}$. Thermal diffusivity is inversely proportional to the gas pressure, so that, based on Table 1, one can write the following estimate for $\chi$ :

$\chi\left(\mathrm{cm}^{2} / \mathrm{s}\right) \approx 150 / p($ torr $)$ 
Using Eq. (3.13) for the thermal energy deposition $q$ per unit length, together with Eqs. (3.21) and (3.22), one can find the energy deposited per unit volume prior to arrival of the subsequent pulse, $q / \pi r_{\text {therm }}^{2}$. Dividing it by the isobaric specific heat per unit volume, $c_{p}$, one finds the on-axis temperature increase prior to arrival of the next pulse: $\Delta T(K) \approx 4.2 \cdot 10^{-4} p($ torr $) \Sigma\left(\mathrm{cm}^{2} / \mathrm{g}\right) W(\mathrm{~mJ})$

The plot of $\Delta T$ vs pressure for $W=2 \mathrm{~mJ}$ and several beam energies is presented in Fig. 3.7. The same figure shows also the radius of the heated channel by the time of the arrival of the next pulse. Obviously, only the domain where this radius is less than the tube radius $b=7 \mathrm{~cm}$ is physically meaningful. When the radius becomes formally larger than the tube radius, heat loss to the massive tube walls begin, and the temperature drops below the estimate (3.23), becoming too low to cause any significant effects.

These estimates will be used in Sec. 5.1 to evaluate distortions of the wave front associated with the density non-uniformity associated with temperature non-uniformity.

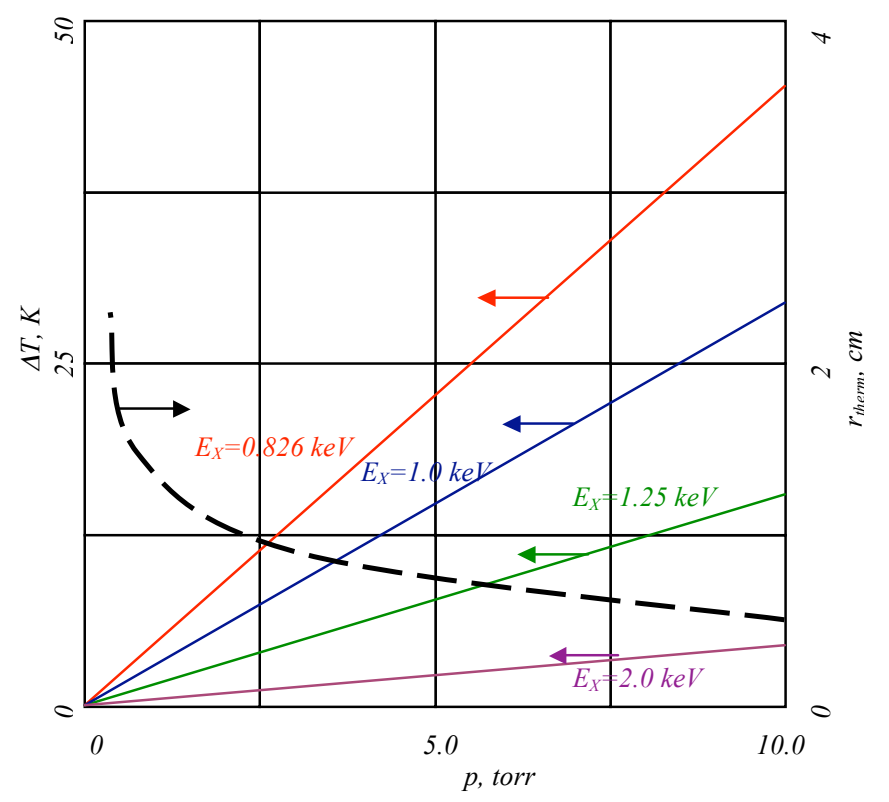

Fig. 3.7 Temperature increase on axis just before arrival of the next pulse (solid lines, left scale) and the radius of the heated zone $r_{\text {therm }}$ (dashed line, right scale). The beam pulse energy $W=2 \mathrm{~mJ}$, efficiency of heating $\eta=0.5$.

\subsection{Chemical interaction of nitrogen with apertures}

Gas ionization and heating may lead to enhanced chemical interaction with the apertures, especially with the entrance aperture, where the X-ray beam is still unattenuated. The radius of the aperture is $2 \mathrm{~mm}$, so that heated and ionized gas may get into direct contact with it. This effect is strongest at the lower end of beam energies, where the energy deposition in the vicinity of the entrance aperture can be quite high. It is hard to make reliable estimates of chemical processes with participation of nitrogen ions 
and atoms. Their total number produced in the vicinity of the aperture is relatively small, but $\mathrm{CW}$ operation of LCLS may lead to the gradual accumulation of damage in the long run.

These concerns relate to the operation of the LCLS facility at design energy per pulse of $2 \mathrm{~mJ}$. At smaller intensities, all the effects mentioned in Secs. 3.3 and 3.4 become weaker.

3.5 Possible variation of the attenuation vs the beam intensity

As was shown in Sec. 3.3, in the CW mode of operation of LCLS, a column with increased temperature will be formed near the axis. The density in this column will be lower than the initial one, due to the radial pressure balance. The density depression is largest near the axis, where it is equal to $\Delta \rho=-\rho \Delta T / T$, with $\Delta T$ determined by Eq. (3.23). There will be the corresponding decrease in the attenuation coefficient near the axis. When evaluating this decrease, one should remember that the heating is nonuniform along the axis, being largest near the entrance and smallest near the exit. As the density depression in a particular axial position is proportional to the pulse energy at this position, one can easily show that

$\frac{\Delta A}{A \ln A}=\int_{0}^{L} \frac{\Delta \rho}{\rho L} d z=-\frac{\Delta T_{0}}{T L} \int_{0}^{L} e^{-z / l_{a b s}} d z=-\frac{\Delta T_{0} l_{a b s}}{T L}\left(1-e^{-L / l_{a b s}}\right)$,

so that

$\frac{\Delta A}{A}=-\frac{\Delta T_{0}}{T}\left(1-\frac{1}{A}\right)$.

According to Fig. 3.7, the relative decrease of the attenuation coefficient is largest at the lower energies of X-ray photons: at the energies $E_{X}<1.5 \mathrm{keV}$ it can be as large as a few percent. On the other hand, effect of this magnitude corresponds to the highest intensity ( $2 \mathrm{~mJ}$ per pulse). For lower intensities it will be proportionally weaker. Also, this is a systematic effect, not varying from pulse to pulse, if the pulse energy $W$ is held constant.

As was mentioned in Sec. 3.3, thermal convection induced by the temperature non-uniformity is too weak to enhance the spreading of the heat over the cross-section. The spreading could be enhanced by introducing a strong forced convection, with velocities exceeding $1 \mathrm{~m} / \mathrm{s}$.

\subsection{Head-to-tail variation}

When the next pulse of X-rays enters the gas attenuator, it "sees" the medium that has experienced relaxation processes after the passage of the previous pulse. In particular, the ionization state of the medium has reached its "average" value. During the pulse, the front part of the beam propagates through this relaxed medium, which it perturbs, so that the rear part propagates through, strictly speaking, somewhat changed medium. Accordingly, attenuation of the rear part may be different from the attenuation of the front part.

Kinetics of fast processes occurring in the gas within the pulse duration $\sim 300 \mathrm{fs}$, has been considered in Ref. [11] and the conclusion was drawn that secondary ionizations 
cannot occur at this timescale. Likewise, no redistribution of molecules or ions over the beam radius may occur. So, the only process that has to be taken in the context of headto-tail variations is the primary ionization.

As the beam absorption occurs primarily via the photo-ionization, the number of ions created per unit volume near the beam axis during the whole beam pulse can be found by dividing the expression (3.17) with $\eta=1$ by the energy of one X-ray photon $E_{X}$, so that

$$
n_{i}^{(1)}=\frac{2 \rho_{0} \Sigma W}{\pi w^{2} E_{X}}
$$

where the superscript " 1 " indicates that we consider only "primary" ions. This number is to be compared with the number density of neutral atoms in the gas $n_{0}=\rho_{0} / M_{N}$, where $M_{N}$ is atomic mass of the nitrogen atom. Numerically, one has:

$$
\frac{n_{i}^{(1)}}{n_{0}}=9.2 \cdot 10^{-3} \frac{\Sigma\left(\mathrm{cm}^{2} / \mathrm{g}\right) W(\mathrm{~mJ})}{[w(\mu \mathrm{m})]^{2} E_{X}(\mathrm{keV})}
$$

The highest degree of the primary photo-ionization corresponds to the lowest photon energy $(0.826 \mathrm{keV})$, because of a very large cross-section $\Sigma$. Substituting $W=2 \mathrm{~mJ}, w=957$ $\mu \mathrm{m}$, and the tabular value for $\Sigma$, one finds that even at the lowest photon energy of 0.826 $\mathrm{keV}$ the ratio $n_{i}^{(1)} / n_{0}$ is very small, $\sim 7 \cdot 10^{-5}$. Respectively, one should not expect any significant variation of the attenuation coefficient between head and tail of the pulse.

\section{Thermo-mechanical effects in the solid attenuator}

\subsection{Thermal expansion and thermal stresses}

As the solid attenuator is an inseparable part of the whole attenuation system, we present here a brief discussion of the effects that are caused by the energy deposition to beryllium foils (slabs) of which the solid attenuator is made. The thickness of the slabs is approximately, 10, 100, 500, 1000, 2000, 4000, 8000, and $16000 \mu \mathrm{m}$. We assume that, in the initial state, the material is uniform and the slab surfaces are planar and parallel to each other.

When the X-ray pulse hits the beryllium foil, it deposits its energy into it, mostly via the photoabsorption process. There is a head-to-tail effect of the same nature as that discussed in Sec. 3.4; as was in the case of the gas attenuator, it is negligibly small. Any deformation of the foil during the X-ray pulse is negligibly small (indeed, the acoustic crossing time for a $10 \mu \mathrm{m}$ thick beryllium foil is $\sim 2 \mathrm{~ns}$, which has to be compared with a few hundred femtosecond X-ray pulse width). So, heating during the pulse does not create any problems with the beam attenuation or wave-front distortions. Deformations are negligibly small and, therefore, the planarity of the wave front is not directly affected. We concentrate, therefore, on the processes occurring on much longer time-scale, up to many inter-pulse times.

The photoelectrons disperse their energy by secondary ionizations and excitations, and eventually, within the time of a few picoseconds, the deposited energy is thermalized [12]. As the range of the photoelectrons in Beryllium is typically less than a micron, most of the energy is thermalized in situ. The temperature increase follows the fluence distribution over the beam radius. We assume that the distribution is Gaussian, Eq. (3.15). 


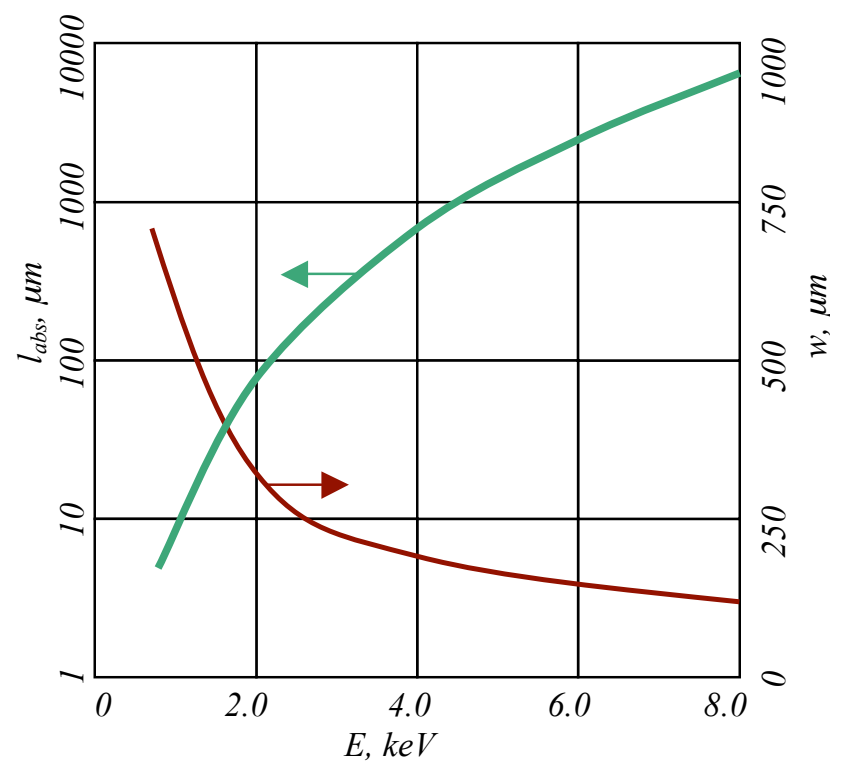

Fig. 4.1 The beam radius $w$ at the location of the solid attenuator (magenta line, right scale) and the e-folding absorption length in beryllium (green line, left scale) vs the beam energy

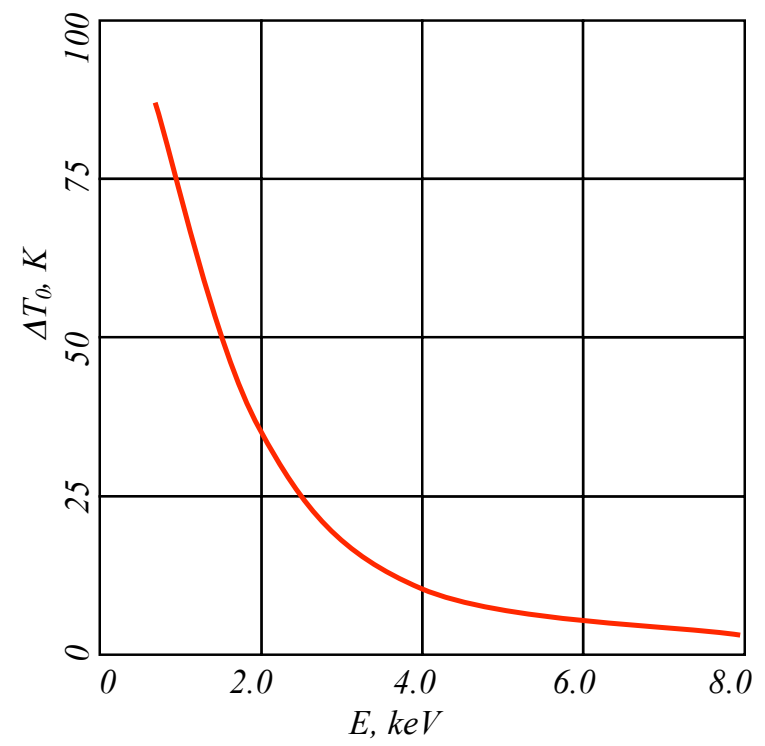

Fig. 4.2 Temperature increase at the surface of beryllium for $2 \mathrm{~mJ} \mathrm{X-ray}$ pulses in the location of the solid attenuator. The beam radius was taken from Table 4. 
The maximum initial temperature increase occurs at the surface, where the beam energy has not been attenuated yet. The energy per unit volume deposited here can be evaluated according to equation (3.17), with the parameter $\eta$ close to 1 :

$Q=\rho_{0} \Sigma F_{0}=\frac{2 \rho_{0} \Sigma W}{\pi w^{2}}=\frac{2 W}{\pi \ell_{a b s} w^{2}}$.

Here $\rho_{0}=1.85 \mathrm{~g} / \mathrm{cm}^{3}$ is the beryllium density, $\Sigma$ is the cross-section per unit of linear mass density and $\ell_{a b s}$ is an e-folding absorption length:

$\ell_{a b s}=\frac{1}{\rho_{0} \Sigma}$.

In "practical" units, one has

$$
Q\left(J / \mathrm{cm}^{3}\right)=6.4 \times 10^{8} \frac{W(\mathrm{~mJ})}{\ell_{a b s}(\mu \mathrm{m})[w(\mu \mathrm{m})]^{2}} .
$$

The width of the beam $w$ at the location of the solid attenuator varies from $957 \mu \mathrm{m}$ at $0.826 \mathrm{keV}$ to $157 \mu \mathrm{m}$ at $8.26 \mathrm{keV}$ [2], Fig. 4.1; this figure contains also the energy dependence of the attenuation length.

Table 3 Thermo-mechanical parameters of beryllium

\begin{tabular}{|c|c|c|c|c|c|c|}
\hline Density, $\rho$ & $\begin{array}{c}\text { Specific } \\
\text { heat, } \mathrm{c}_{\mathrm{p}}\end{array}$ & $\begin{array}{c}\text { Thermal } \\
\text { diffusivity, } \chi\end{array}$ & $\begin{array}{c}\text { Volumetric } \\
\text { thermal exp. } \\
\text { coefficient, } \alpha\end{array}$ & $\begin{array}{c}\text { Young's } \\
\text { Modulus, } E\end{array}$ & $\begin{array}{c}\text { Poisson } \\
\text { Ratio, } \sigma\end{array}$ & $\begin{array}{c}\text { Compressional } \\
\text { sound speed, } s\end{array}$ \\
\hline $1.85 \mathrm{~g} / \mathrm{cm}^{3}$ & $3.3 \mathrm{~J} / \mathrm{cm}^{3} \mathrm{~K}$ & $0.52 \mathrm{~cm}^{2} / \mathrm{s}$ & $3.1 \times 10^{-5}$ & $3 \times 10^{12} \mathrm{erg} / \mathrm{cm}^{3}$ & 0.2 & $1.25 \times 10^{6} \mathrm{~cm} / \mathrm{s}$ \\
\hline
\end{tabular}

Dividing $Q$ by the specific heat (Table 3 ), one finds the maximum temperature increase after the passage of one pulse:

$$
\Delta T_{0}(K)=1.94 \times 10^{8} \frac{W(m J)}{\ell_{a b s}(\mu m)[w(\mu m)]^{2}}
$$

The plot of $\Delta T_{0}$ vs the beam energy is presented in Fig. 4.2. The strongest surface heating occurs at low X-ray energies. Still, it is well below the critical temperature for which mechanical stresses caused by non-uniform thermal expansion exceed the yield strength (Ref. [13]).

After the passage of the X-ray pulse, temperature re-distribution begins: the temperature spreads both laterally and axially. By the time $t$ of the arrival of the next pulse, the temperature spreads over the distance $\sqrt{2 \chi t} \sim 1 \mathrm{~mm}$. We will consider only foils with the thickness $h$ less than $1 \mathrm{~mm}$ : the thicker foils will be used only in the relatively high X-ray energy domain, above $4-5 \mathrm{keV}$, where, according to Fig. 4.2, the heating is very weak. For the foils with $h<1 \mathrm{~mm}$, the further radial broadening of the temperature profile will occur with the temperature having already become uniform in the axial direction, even if the foil thickness is greater than the attenuation length $l_{a b s .}$ When the next pulse arrives, the first one will be already spread over $1 \mathrm{~mm}$ radius. The third pulse will appear on the top of the previous two, etc. A quasi-stationary temperature 

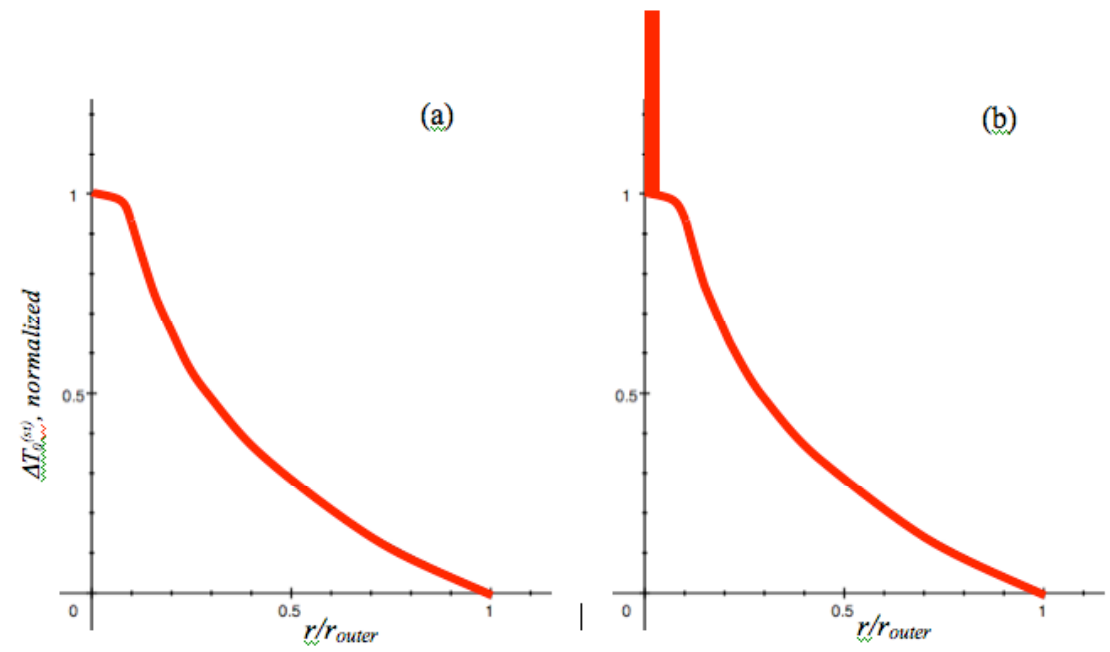

Fig. 4.3 Radial temperature distribution in the beryllium foil (slab): a) just before the arrival of the next pulse; the curve is normalized to the maximum temperature, Eq. (4.6); b) the same just after the arrival of the beam pulse; a large peak appears on the top of a smooth "average" curve; the normalization is the same as in Panel (a).

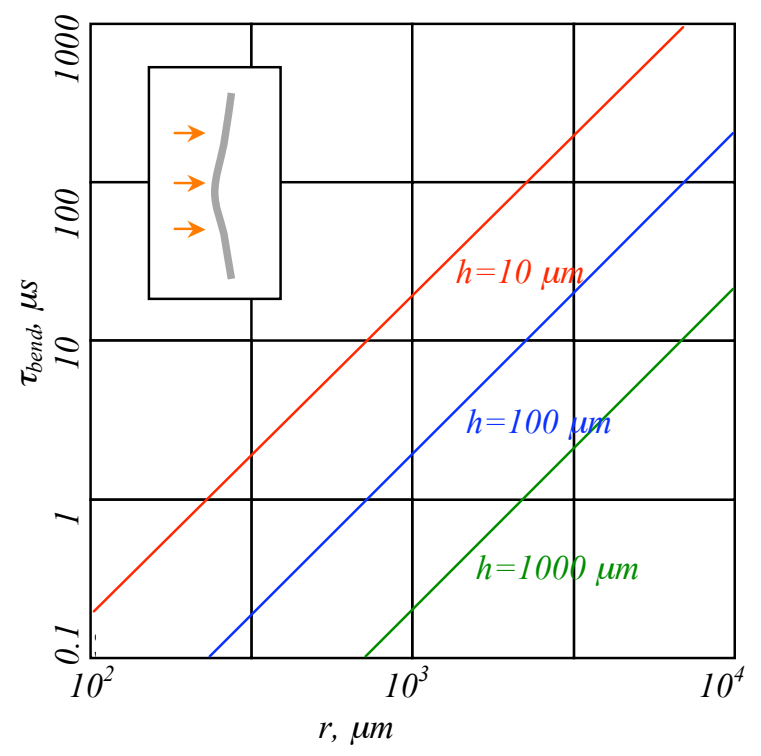

Fig. 4.4 Characteristic time for developing the bending deformation of a beryllium foil or a thin beryllium slab of a thickness $h$ under the action of differential thermal expansion or due to the Euler instability. The deformation of a foil is shown in the inset. The beam direction is shown with the arrows. The magnitude of the deformation is grossly exaggerated. 
distribution will establish when the heat from the first pulse reaches the massive clamp that holds the foil at a radius $r_{\text {outer }} \sim 1 \mathrm{~cm}$. The clamp is not actually circular but, as we will see, the central temperature increase depends on $r_{\text {outer }}$ only logarithmically. This quasi-stationary state will be established within the time $\sim r_{\text {outer }}^{2} / 2 \chi \sim 0.5 \mathrm{~s}$.

The radial dependence of the quasi-stationary component of the temperature at the radial distances from the axis exceeding $1 \mathrm{~mm}$ have a logarithmic profile,

$\Delta T^{(s t)} \approx\left(1-\frac{1}{A_{\text {solid }}}\right) \frac{W}{2 \pi t c_{p} \chi h} \ln \frac{r_{\text {outer }}}{r}, r>1 \mathrm{~mm}$,

where $t=1 / 120 \mathrm{~s}$ is the distance between the pulses, $A_{\text {solid }}$ is the attenuation coefficient in a slab of thickness $h$, and the parameters $c_{p}$ and $\chi$ are given in Table 3 . In the zone $r<1 \mathrm{~mm}$, this dependence switches to a non-singular "cap," as shown in Fig. 4.3 (a) .

On the top of the quasi-stationary temperature distribution (4.5), every 1/120 s appears a sharp peak. So, the overall temperature distribution just after arrival of the Xray pulse looks as shown in Fig. 4.3 (b). The figure 4.3 (a), according to this discussion, corresponds to the instance just before the arrival of the next pulse, when the previous one has already been "absorbed" by a quasi-steady distribution. In a more complete analysis, one would find some waviness superposed on the smooth distribution (4.5); this (small) waviness is caused by the discreteness of the heating pulses.

The maximum in Fig. 4.3 (a) can be evaluated as

$\Delta T_{0}^{(s t)}(K) \approx 300 \frac{W(m J)}{h(\mu m)}$

We assumed here that $A_{\text {solid }}=e=2.718$ and $r_{\text {outer }} / r=10$. For $W=2 \mathrm{~mJ}$ and $h=100 \mu \mathrm{m}$, the quasi-steady component of the temperature increase on axis is $\sim 6 \mathrm{~K}$. The e-folding attenuation length is equal to $h=100 \mu \mathrm{m}$ for the beam energy of approximately $2.2 \mathrm{keV}$.

Table 4 Interaction of 2-mJ X-ray pulses with beryllium

\begin{tabular}{|l|l|l|l|}
\hline$E, \mathrm{keV}$ & $l_{a b s}, \mu m$ & $w, \mu m$ & $\Delta T_{0}, K$ \\
\hline 0.826 & 5.13 & 957 & 82.5 \\
\hline 2.0 & 72.5 & 387 & 35.7 \\
\hline 4.0 & 629 & 256 & 9.4 \\
\hline 6.0 & 2237 & 200 & 4.3 \\
\hline 8.26 & 5775 & 157 & 2.7 \\
\hline
\end{tabular}

\subsection{Effect of thermal expansion on the beam attenuation}

In this section we assume that no warping or buckling occurs and that the only effect of the thermal expansion is the change of the line density. We concentrate on the foils/slabs of a thickness of less than $1 \mathrm{~mm}$, as the thicker ones will be used only at higher $\mathrm{X}$-ray energies, where thermal effects are minimal (we briefly discuss these thicker slabs at the end of this section). As has been mentioned in Sec. 4.1, the temperature prior to arrival of the next pulse, depends only on radius, Fig. 4.3 (a). The density variation is $\Delta \rho / \rho=-\alpha \Delta T$, where $\alpha$ is a volumetric thermal expansion coefficient, Table 3 . The thickness of the foil (slab) increases as $\Delta h / h=(\alpha / 3) \Delta T$, where $\alpha / 3$ is a linear thermal expansion coefficient. Therefore, the line density on axis decreases by 
$\frac{\Delta(\rho h)}{\rho h}=-\frac{2 \alpha \Delta T_{0}^{(s t)}}{3}$

Accordingly, the attenuation coefficient $A$ decreases by

$\frac{\Delta A_{\text {solid }}}{A_{\text {solid }}}=-\frac{2 \alpha \Delta T_{0}^{(s t)}}{3} \ln A_{\text {solid }}$,

where $\Delta T_{0}^{(s t)}$ is determined by Eq. 4.6. As the thermal expansion coefficient of beryllium is quite low, this effect is weak, with the ratio $\Delta A_{\text {solid }} / A_{\text {solid }}$ being less than $10^{-3}$ for all the situations of practical interest.

For thicker slabs, which will be used at higher energies of $\mathrm{X}$ rays, the temperature pattern will be different, with a significant axial variation of the time-averaged temperature. This is due to the fact that the time required for the axial heat diffusion in thicker slabs is greater than the inter-pulse time $t$. On the other hand, the temperature increase will be smaller than for the lower-energy X-rays, due to much smaller absorption coefficient. Related variation in the attenuation coefficient will be much smaller than for the lower-energy X-rays.

4.3 Possible gross deformations of slabs caused by thermal stresses

The time within which the temperature redistribution over the distance $r$ occurs can be evaluated as

$\tau_{\text {therm }} \sim \frac{r^{2}}{2 \chi}$

where $\chi$ is the thermal diffusivity given in Table 3 . In "practical" units,

$\tau(s) \sim 10^{-8}[r(\mu m)]^{2}$.

It is of order of $1 \mu \mathrm{s}$ for $r=10 \mu \mathrm{m}$ (that corresponds to the thickness of the thinnest foil); for $r=1 \mathrm{~mm}$ it is $\sim 1 / 120 \mathrm{~s}$; for $r=1 \mathrm{~cm}$ it is of order of one second.

The other important characteristic time is the sound propagation time over a distance $r$

$\tau \sim \frac{r}{s}$,

where $s$ is a sound speed for longitudinal sound waves. It is much shorter than the pulse separation time even for the largest distances involved in our problem. Similar propagation time corresponds to transverse acoustic waves with the displacement vector lying in the plane of the foil.

The third characteristic time is the propagation time for the waves with the displacement vector perpendicular to the foil surface. In the case where the foil thickness $h$ is small, the phase velocity of these waves is much smaller than the sound speed [14]. Specifically, in the case where $h<\lambda / 2 \pi$, with $\lambda$ being the wavelength of these "bending" oscillations, their phase velocity is [14]:

$\mathrm{v}_{\text {bend }}=\frac{2 \pi}{\lambda} \sqrt{\frac{h^{2} E}{3 \rho\left(1-\sigma^{2}\right)}} \sim \frac{h}{\lambda} s<s$,

where $E$ is the Young's modulus and $\sigma$ is the Poisson ratio for beryllium. The time for this wave to propagate a distance of order of the half of the wave-length, is 
$\tau_{\text {bend }}=\lambda / 2 \mathrm{v}_{\text {bend }}$; if we identify $\lambda / 2$ with the diameter of the heated spot $2 r$, we find a characteristic time of the bending deformation, which can be caused by the non-uniform thermal expansion or by the buckling instability of a centrally heated and clamped thin disc (see below). We find from Eq. (4.12):

$\tau_{\text {bend }} \sim \frac{4 r^{2}}{\pi} \sqrt{\frac{3 \rho\left(1-\sigma^{2}\right)}{h^{2} E}}$

This time is plotted in Fig. 4.4 as a function of $r$ for several thicknesses of the beryllium slab (foil). We concentrate here on relatively thin foils, $h<1 \mathrm{~mm}$; the thicker ones would be used for attenuating high-energy pulses, where the heating is weak (see Fig. 4.2) and the thermo-mechanical effects are unimportant.

If the foil is non-uniformly heated over the thickness in a spot of a radius $r$, and the time $\tau_{\text {bend }}$ is shorter than the temperature equilibration time, the foil will be deformed as shown in Fig. 4.5, due to the stronger expansion near the surface facing the undulator. This deformation decreases later in time due to the temperature equilibration over the foil thickness, and the foil returns to its initial state, just to repeat the whole cycle after the next pulse.

For the thinnest foils of 10 and $100 \mu \mathrm{m}$, the quasi-steady temperature increase may cause the onset of a "buckling" (Euler) instability: as the foil is clamped on the outer radius, it cannot expand radially, this giving rise to a radial stress which may cause the buckling; for thin foils, the corresponding instability requires a small stress, that scales as $\left(h / r_{\text {outer }}\right)^{2}[14]$ and can be exceeded even for relatively small temperature increase. We leave the solution of the corresponding complex problem of the elasticity theory for the possible future work and here limit ourselves to the suggestion that the foils of 10 and $100 \mu \mathrm{m}$ thickness be used for the lower end of the beam energies $(<2.5 \mathrm{keV})$ only if absolutely necessary. We note also that, for smaller pulse energies, below $0.1-0.2 \mathrm{~mJ}$, the temperature increase becomes small even at the lower end of the beam energy range $(<2.5 \mathrm{keV})$.

\section{Effect of the attenuator on the wave front}

\section{1 Effect of the gas attenuator}

The effect of the gas attenuator on the wave front is associated with possible nonuniformities of the density distribution within the beam aperture. In particular, the beam heating of the gas and resulting density depression near the axis of the attenuator could lead to some focusing of the beam, whereas possible up-down asymmetries could lead to a beam deflection. The presence of the turbulent density fluctuations in the nozzle formed by the entrance aperture could cause some beam scattering. We will see, however, that all these factors are small and lead to deformation of the wave-front in the range of a fraction of nano-radian.

With regard to the thermally-driven density variations, their magnitude is significantly reduced by both conductive and convective temperature spread between the pulses, whereas the role of turbulent fluctuations is small because they are localized in a short segment of the flow near the entrance aperture.

We use a standard representation of the refraction index $N_{r}$ (see Ref. [8]): 
$N_{r}=1-\delta-i \beta$

where the coefficients $\delta$ and $\beta$ are both small compared to unity; they both are

proportional to the gas density. The spatial scale of variation of the coefficients in $N_{r}$ is very large compared to the wavelength of X-rays, so that the ray description can be used. We orient the Cartesian coordinate frame so that the axis $z$ is parallel to the axis of a nondisturbed beam, axis $y$ corresponds to the vertical direction and axis $x$ to the horizontal direction. By using standard equations of refraction in non-uniform media (e.g., Ref. [17]), one obtains the following expression for the deflection angle of a ray:

$\vartheta_{y}=-\frac{\delta}{n} \frac{\partial}{\partial y} \int n d z ; \vartheta_{x}=-\frac{\delta}{n} \frac{\partial}{\partial x} \int n d z$

To get some sense of the possible magnitude of the effect, we consider the situation where there is a temperature difference $\Delta T$ between the lower and the upper part of the attenuator tube. Then, there will be a density variation $\Delta n=n_{0} \Delta T / T_{0}$ on the scale of $2 b$, so that

$$
\vartheta_{y} \approx-\delta \frac{\Delta T}{T_{0}} \frac{L_{0}}{2 b}
$$

On the other hand, the attenuation coefficient $A$ is related to the parameter $\beta$ in Eq. (5.1) by

$$
\ln A=2 L_{0} \beta \frac{2 \pi}{\lambda_{X}}
$$

where $\lambda_{\mathrm{x}}$ is the wave-length of x-rays. The factor " 2 " in front of $L_{0}$ reflects the fact that $A$ is the attenuation of the intensity (not of the amplitude). Eliminating $L_{0}$ from Eqs. (5.3) and (5.4), we obtain a convenient representation for the deflection angle:

$$
\vartheta_{y} \approx-\frac{\delta}{\beta} \frac{\Delta T}{T_{0}} \frac{\lambda_{X} \ln A}{8 \pi b}
$$

One can equivalently express this result in terms of the coefficients $f_{1}$ and $f_{2}$ from Ref. [8], as it was done in Ref. [4]. The strongest deviation would correspond to a $2.5 \mathrm{keV}$ beam (Table 5). But making even a grossly exaggerated estimate of the possible up-down temperature difference of $\Delta T \sim 1 \mathrm{~K}$ (that might appear if the temperature control were off), one finds that the deflection angle is less than $0.1 \mathrm{nrad}$, i.e. negligible. The ratio $\lambda_{X} \delta / \beta$ is plotted vs the X-ray energy $E_{X}$ in Fig. 5.1, both for nitrogen and beryllium.

One more source of distortion of the wave front is the radial density variation produced by beam heating of the gas and solid attenuator and discussed in Secs. 3.3 and 4.2. As the density is smaller on axis, this type of density non-uniformity will act as a focusing lens (we remind that the refraction index for X-rays is less than 1 and increases with the decreasing density).

The density profile within the beam aperture can be approximated as a parabola,

$$
\Delta n=-n \frac{\Delta T_{0}}{T}\left(1+\frac{r^{2}}{r_{\text {therm }}^{2}}\right),
$$

with $r_{\text {therm }}$ defined by Eq. (3.21). Using Eqs. (5.2) and noting that the radial density derivative for parabolic profile is proportional to $r$, one finds the following expression for the focal length:

$$
f=\frac{T}{\Delta T_{0}} \frac{\beta}{\delta} \frac{2 \pi r_{\text {therm }}^{2}}{\lambda_{X}} \frac{A}{A-1}
$$


Table 5 Deflection angle Eq. (5.5), for $b=7 \mathrm{~cm}, \Delta T / T_{0}=1 / 300$, and $\ln A=1(A=2.7183)$

\begin{tabular}{|c|c|c|c|}
\hline X-ray energy, keV & $\begin{array}{c}\text { Wavelength } \lambda_{X}, \\
\text { Angstrom }\end{array}$ & $\delta / \beta$ & $\begin{array}{c}\text { Deflection angle, } \\
\text { prad }\end{array}$ \\
\hline 0.826 & 15.02 & 4.81 & 13.7 \\
\hline 1.00 & 12.41 & 6.68 & 15.75 \\
\hline 1.25 & 9.93 & 9.81 & 18.51 \\
\hline 1.50 & 8.27 & 13.58 & 21.3 \\
\hline 1.75 & 7.09 & 17.95 & 24.2 \\
\hline 2.00 & 6.205 & 22.99 & 27.1 \\
\hline 2.25 & 5.52 & 28.67 & 30.06 \\
\hline 2.50 & 4.96 & 35.03 & 33.01 \\
\hline
\end{tabular}

The shortest focal length corresponds to low photon energies and highest gas pressures. However, even here the focal length is incredibly large, $\sim 100 \mathrm{~km}$. So, this source of the wave front distortion is also negligible.

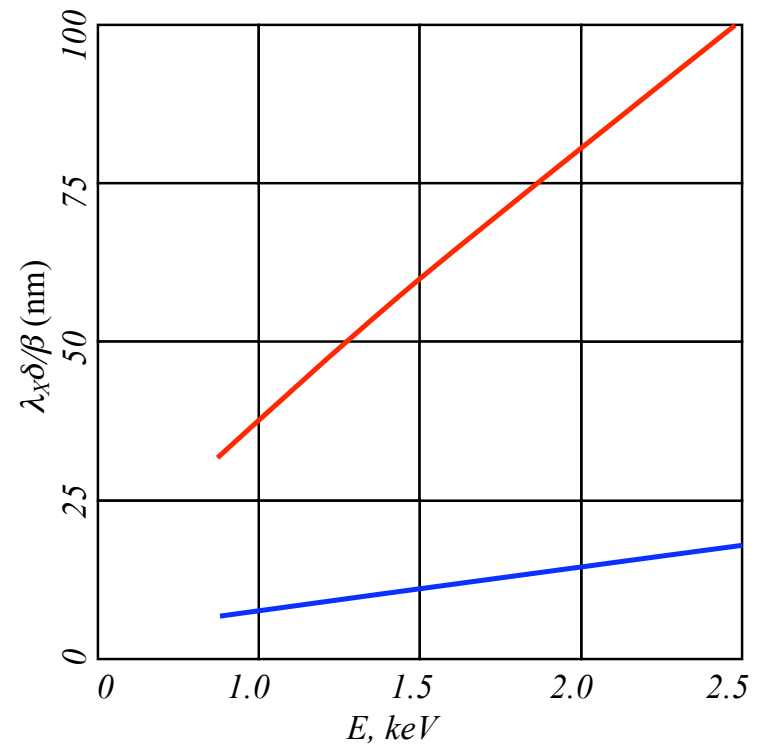

Fig. 5.1 The parameter $\lambda_{X} / \delta / \beta$ that determines the focusing properties of nonuniform nitrogen (blue curve) and beryllium (red curve). The focal length of a lens formed due to the temperature increase near the beam axis is inversely proportional to this parameter.

\section{2 Effect of the solid attnuator.}

The same analysis can be applied to the solid attenuator. The effect is again strongest at lower energies. Assuming that we deal with foils/slabs thinner than $1 \mathrm{~mm}$, we use the model of the axially-uniform temperature and apply Eq. (4.6) for the temperature increase and model (4.7) for the line-density variation. We obtain, instead of Eq. (5.7), $f=\frac{3}{2 \alpha \Delta T_{0}} \frac{\beta}{\delta} \frac{2 \pi r_{\text {therm }}^{2}}{\lambda_{X} \ln A}$ 
Here the focal length becomes somewhat shorter due to the much smaller ratio of beta/delta (Fig. 5.1). This effect is, however, partially neutralized by the smaller thermal expansion effect. The distortion of the wave front is therefore small, unless gross deformations are produced by warping of the foil. In our assessment we assumed that the slab in the initial state is uniform.

\section{Electromagnetic wake}

Intense X-ray pulse propagating through the gas will generate electromagnetic wake, with wavelengths of order of a few centimeters [19]. This wake can, in principle, be used to evaluate the energy $W_{0}$ of the X-ray pulse in future devices; its potential adverse effect is the generation of spurious EM signals in various gauges and detectors. The wake is generated by the evolving charge distribution produced after the passage of the X-ray pulse and is related to the fact that the X-reay beam in the LCLS facility will be linearly polarized.

We first consider this effect for relatively low pressures, where the electrostatic confinement of the primary photoelectrons is absent and they move freely in the radial direction (until they scatter on the gas molecules or hit the wall). The angular distribution of the photoelectrons [20] in the plane perpendicular to the beam axis has a form of two lobes oriented along the plane in which the electric field of the wave lies (Fig. 6.1). The range of the photoelectrons at low pressures that we consider now is comparable to or larger than the attenuator radius. So, they propagate freely away from the axis and form the space charge distribution shown in Fig. 6.2. Note that these electrons are nonrelativistic, so that the electrostatic approximation can be used to describe the electric field distribution inside the tube. This is correct everywhere except for the locations very near to the x-ray bunch, at the distances less than a couple of tube radii behind it. In this zone the delay effect is significant. We, however, focus on what happens at a distance of a couple or more tube radii behind the bunch, at the time-scales exceeding, roughly, 0.5 ns (that is, $2 b / c$ ) after the X-ray pulse passing.

The charge distribution that is formed is of a quadrupole nature (Fig. 6.2), with the characteristic size of the quadrupole $\sim \mathrm{v}_{0} t / 2$, where $\mathrm{v}_{\mathrm{o}}$ is the velocity of the photoelectrons and $t$ is the time after passing of the X-ray pulse through the cross-section in question. A surface charge induced on the surface of the attenuator pipe is also shown. For the electric field strength on the wall we have approximately

$$
E \approx \frac{q}{b}\left(\frac{\mathrm{v}_{0} t}{2 b}\right)^{2} \cos (2 \vartheta)
$$

where $q$ is the ion charge per unit length and $\theta$ is the azimuthal angle measured from the polarization plane (Fig. 6.1). With the photoelectrons escaping to the walls, the field structure will become more and more axisymmetric (monopolar, produced by the ion kernel at the axis). After the photoelectrons reach the wall (i.e., at $t \sim 2 b / \mathrm{v}_{0}$ ) and are absorbed by it, the field reaches a quasi-steady value of $E_{\max } \sim q / b$.

The number of primary ionizations produced by the X-ray pulse per unit length can be evaluated as

$$
\frac{\partial N_{i}^{(1)}}{\partial x}=\frac{W \Sigma \rho}{E_{X}},
$$




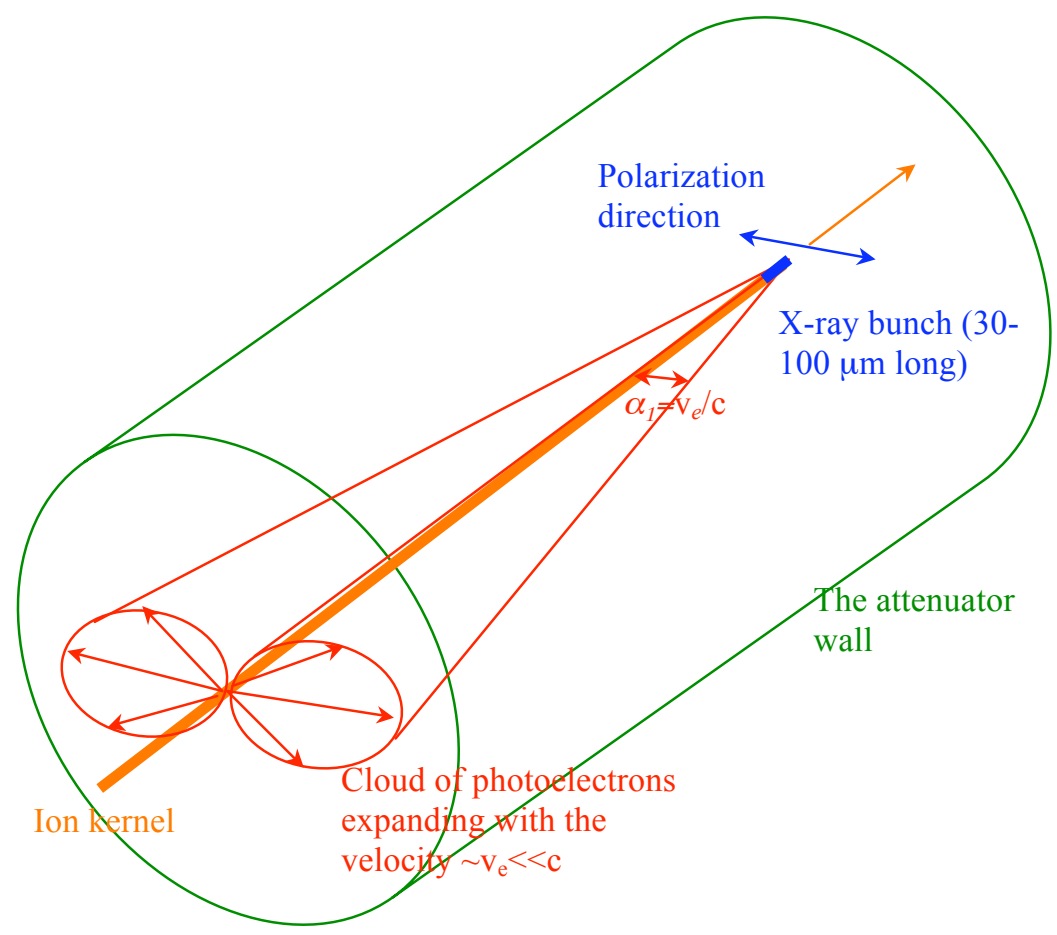

Fig. 6.1 Distribution of the expanding electron cloud produced by the polarized $\mathrm{X}$-ray pulse.

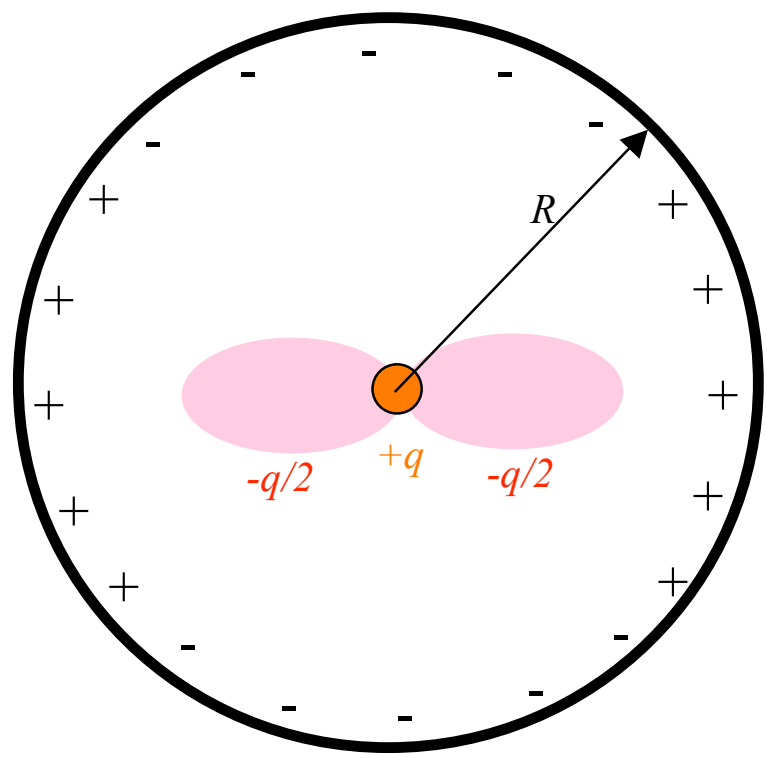

Fig. 6.2 Charge distribution at the moment when photo-electrons are half-way to the walls (i.e., at $\sim 8 \mathrm{~ns}$ for the $0.826 \mathrm{keV}$ pulse and $\sim 2 \mathrm{~ns}$ for the $8.26 \mathrm{keV}$ pulse.

Instantaneous surface charge distribution on the walls is also shown. 
so that

$$
q=e \frac{\partial N_{i}^{(1)}}{\partial x} .
$$

Substituting this into expression $E_{\max } \sim q / b$, one finds the following numerical estimate for the maximum field:

$$
E_{\max }(V / \mathrm{cm}) \sim 0.2 \frac{W(\mathrm{~mJ}) \Sigma\left(\mathrm{cm}^{2} / \mathrm{g}\right) \mathrm{p}(\text { torr })}{E_{X}(\mathrm{keV})} .
$$

Taking the pressure to be $1 / 3$ of that at which space-charge effects come into play, i.e., multiplying the critical pressure in Eq. (3.14) by 1/3, and substituting it into Eq. (6.4), one finds:

$E_{\max }(\mathrm{V} / \mathrm{cm}) \sim 100\left[E_{X}(\mathrm{keV})-0.4\right]$

$\left(p=p_{\text {el-stat }} / 3\right)$. The range of photoelectrons at the pressures below $p_{\text {el-stat }}$ is greater than the tube radius $b$, so that their interaction with the gas will not have a strong effect on the dynamics of the electric field.

As one can see, the magnitude of the electric field at sub-critical pressures will be on the order of $100-200 \mathrm{~V} / \mathrm{cm}$.

In the regime of a strong electrostatic confinement, the maximum electric field on the wall will be smaller than $q / r$, because most of the photoelectrons will be turned back to the axis well before they reach the wall; an amount that would reach the wall would correspond to the critical amount required to create a potential difference of order of the photoelectron energy. In other words, the maximum electric field at the wall will remain roughly the same as at the critical pressure (3.14).

Another effect that will come into play at higher pressures will be secondary ionizations and generation of a denser neutralizing plasma which will eventually shield the "primary" space charge and thereby reduce the effect. We note, however, that, for the pressures below $1 / 3$ of the critical pressure, the secondary ionization is relatively insignificant [21].

The ion kernel will expand radially, experiencing charge-exchange and elastic interactions with the gas. At the pressures below 0.1 torr, it will reach the wall and disappear there before the arrival of the next pulse. At higher pressures, its diffusion will take time exceeding $0.01 \mathrm{~s}$, and some average (over many pulses) ion density will be established. These ions will be neutralized by cold electrons and gradually recombine. Details of these processes have been discussed in the context of the gas detector in Ref. [10].

\section{Calibration of the gas attenuator relative to the solid attenuator}

As has been mentioned in Sec. 2.2, there exists several sources of uncertainties in the a priori evaluation of the beam attenuation. They include, in particular, our imperfect knowledge of the relevant cross-sections, as well as possible errors in the measurements of the gas temperature. To provide a cross-check of the predicted vs real attenuations, one can calibrate the gaseous attenuator with respect to the solid attenuator. The calibration will be based on the use of the gas detector downstream of the attenuator.

Inserting one of the attenuation blocks of the solid attenuator and keeping the gas attenuator empty, one will get some signal from the gas detector. After that, removing the solid attenuator block and gradually increasing the pressure in the gas attenuator, one 
would reach the situation where the signal from the gas detector will become the same. This exercise does not require any calibrations of the gas detector. What is required, is stability of the X-ray pulses (their energy content and photon energy) and sufficiently high signal-to-noise level from the gas detector. One would then compare the pressure in the gas attenuator with that predicted by Eq. (2.2), assuming that the solid attenuator yields a predictable attenuation. Possible disagreement would characterize the actual accuracy achievable with the attenuation system.

\section{Summary and discussion}

We have considered a variety of effects that determine the performance of the gas attenuator and solid attenuator. The overall conclusion is that this system will become a convenient and reliable tool for the experiments on LCLS.

At the early stage of operations, various cross checks are advisable, which would eliminate possible uncertainties and oversights. In particular, the comparison of the attenuation produced by the gas attenuator alone and the solid attenuator alone, could be one of such tests. It would include the use of a downstream gas detector, but only in the null-mode, so that the results will not depend on the subtleties affecting the performance of the gas detector. It might also be helpful to study transients in the response of the gas pressure to the rapid change in the gas influx.

Among the factors that may cause deviation of the attenuation coefficient from the "dialed in" value, is the insufficient accuracy of the photo-absorption cross-sections of Ref. [8]. According to an expert opinion [18], these uncertainties may be as large as a few percent at lower energies. Various cross-checks with the use of both gas and solid attenuators would be quite helpful in identifying and evaluating the real scale of this problem.

Heating of the gas near the axis of the device causes density decrease in this zone and the corresponding decrease of the attenuation coefficient with respect to the "dialed in" value. The effect is maximum at low X-ray energies and highest beam intensities, where it can be as high as 10\%. This effect is a systematic effect and can, in principle, be accounted for by alternative use of a gas and solid attenuator.

We have identified and evaluated several interesting effects that may show up during the normal operation. One of them is formation of a very hot, short-lived gas channel near the entrance aperture of the gas attenuator. It may produce bright flashes of visible light suitable for detection. The other is generation of electromagnetic wakes caused by transient space-charge effects during the propagation of the $\mathrm{x}$-ray pulse through the gas. These wake-fields are relatively weak and will probably not cause any particular problems with the electronics. They potentially can be of some interest as a possible way of detecting X-tray pulses and their energy.

We looked also into the issues of possible damage to the attenuator caused by intense X-ray pulses. In the case of a gas attenuator this may be a long-term degradation of the apertures caused by the chemical erosion. The magnitude of this effect is hard to estimate, but it will certainly become weaker if the gas densities are reduced. This effect, if present, will be most pronounced for the inner side (facing the attenuator) of the entrance aperture of the gas attenuator: the gas pressure is higher there than at the outer side. Periodic inspections of the entrance aperture at the early stages of operation may 
give the necessary confidence that there are no long-term problems. Using existing ports for this purpose could be helpful.

For the solid attenuator, the damage to the foils/slabs may happen only at lower $\mathrm{X}$-ray energies, below $\sim 1.5 \mathrm{keV}$, and highest pulse energies ( $2 \mathrm{~mJ})$. It would be caused by differential thermal expansion and possible warping of these foils. These problems can be avoided if one does not use solid attenuator at these low X-ray energies and uses here only the gas attenuator.

The whole surface of the attenuator will be continuously "showered" by photons produced by the Compton scattering from the gas and/or solid attenuator. The average intensity of this component of radiation will be low, and only very long-term effects of radiation degradation can be expected, mostly in the pressure and temperature gauges and, possibly, in optical components. This is, again, an effect whose magnitude is hard to evaluate in advance, based on first principles.

Distortions of the wave-front of the beam in the gas attenuator turn out to be quite small. They are caused mostly by the variation of the density near the axis and the associated focusing of the beam.

Our results can be used in the analysis and design of similar devices in future Xray generators. 


\section{References}

1. Linac Coherent Light Source (LCLS) SLAC Design Study Report No. SLAC-R521, 1998, National Technical Information Services, 5285 Port Royal Road, Springfield, Virginia, 22161.

2. R.M. Bionta. "Controlling Dose to Low Z Solids at LCLS." LCLS Technical note TN-00-03, January 2000.

3. D. Ryutov, A. Toor. "X-ray attenuation cell." LCLS-TN-00-10, June 2000.

4. D.D. Ryutov, R.M. Bionta, M.A. Mc Kernan, S. Shen, J.W. Trent. "The Physics Analysis of a Gas Attenuator with Argon as a Working Gas." LCLS Technical Note TN-06-1, 2006.

5. S. Shen. Viewgraphs "LCLS-XTOD Gas Attenuator" presented at the LCLS FAC Meeting (October 27, 2005); UCRL-PRES-21558.

6. D.D. Ryutov. "The Gas Flow from the Gas Attenuator to the Beam Line." LCLS Technical Note TN-06-10, 2006.

7. D.D. Ryutov, R.M. Bionta, S.P. Hau-Riege, K.I. Kishiyama, M.D. Roeben, S. Shen. "Operating the LCLS gas attenuator and gas detector system with apertures of 6 mm diameter." LCLS Technical Note TN-08-10, 2008.

8. B.L. Henke, E.M. Gullikson, and J.C. Davis, Atom.Data Nucl. Data Tab. 54, 181 (1993).

9. L.D. Landau, E.M. Lifshitz. "Fluid Mechanics", Pergamon Press, NY, 1987.

10. D.D. Ryutov, S. Hau-Riege, R.M. Bionta. "Space-charge effects in a gas detector.” UCRL-TR-234930; LCLS-TN-07-11, September, 2007.

11. S.P. Hau-Riege, R.M. Bionta, D.D. Ryutov, J. Krzywinski. "Measurement of freeelectron-laser pulse energies by photoluminescence in nitrogen gas." Journal of Applied Physics, 103, 053306, 2008.

12. R.A. London, R.M. Bionta, R.O. Tatchyn, S. Roesler. "Computattional Simulations of High-Intensity X-Ray Matter Interaction.” In: Proceedings of SPIE (R.O. Tatchyn, A.K. Freund, T. Matsushita-Eds), v. 4500, p. 51 (2001).

13. D.D. Ryutov. "Thermal stresses in the reflective X-ray optics for the Linac Coherent Light Source.” Rev. Sci. Instr. 74, 3722 (2003).

14. L.D. Landau, E.M. Lifshitz. "Theory of elasticity", Pergamon Press, NY, 1987.

15. Handbook of Chemistry and Physics, $89^{\text {th }}$ Edition, 2008-2009, Section 6, http://www.hbcpnetbase.com/?BookID=34, CRC (Taylor\&Francis Group).

16. I.K. Kikoin (Ed). "Tables of Physical Quantities" (Moscow, Atomizdat, 1976), in Russian.

17. M. Born. "Principles of optics: electromagnetic theory of propagation, interference and diffraction of light." NY, Cambridge University Press, 1999.

18. E.M. Gullikson. Private communication to D.D. Ryutov, December 2005.

19. D.D. Ryutov. "Electromagnetic perturbation produced by an LCLS X-ray pulse propagating through a gas." LCLS XTOD Group Meeting, October 22, 2007, UCRL-PRES-235430.

20. James H Scofield. "Angular distribution of photoelectrons from polarized Xrays.” Phys. Scr. 41 59-62 (1990).

21. J.L. Barrett and P.B. Hays "Spatial distribution of energy deposited in nitrogen by electrons.” J. Chem. Phys., 64, 743 (1976). 